Research paper

\title{
An algorithm for computing non-concave multifractal spectra using the $S^{v}$ spaces
}

\author{
Thomas Kleyntssens ${ }^{\mathrm{a}}$, Céline Esser ${ }^{\mathrm{b}}$, Samuel Nicolay ${ }^{\mathrm{a}, *}$ \\ a University of Liège, Department of Mathematics, Quartier Polytech 1, allée de la Découverte 12, Bâtiment B37, B-4000 Liège \\ (Sart-Tilman), Belgium \\ ${ }^{\mathrm{b}}$ University of Lille 1, Laboratoire Paul Painlevé, Cité Scientifique, F-59655 Villeneuve d'Ascq, France
}

\section{A R T I C L E I N F O}

\section{Article history:}

Received 29 March 2017

Revised 23 August 2017

Accepted 30 August 2017

Available online 1 September 2017

\section{Keywords:}

Non-concave spectrum

Multifractal formalism

Wavelet

$S^{v}$ spaces

\begin{abstract}
A B S T R A C T
We present an implementation of a multifractal formalism based on the $S^{v}$ spaces and show that it effectively gives the right Hölder spectrum in numerous cases. In particular, it allows to recover non-concave spectra, where other multifractal formalisms only lead to the concave hull of the spectra.
\end{abstract}

(c) 2017 Elsevier B.V. All rights reserved.

\section{Introduction}

Multifractal signals are very erratic signals present in many practical situations such as turbulence [1], physiology [2], biology [3], geoscience [4]. The aim of multifractal analysis is to analyze these signals, whose regularity can change widely from a point to another. Information concerning the global smoothness of a signals can be grasped via its Hölder spectrum, which relies on the Hölder spaces. Such spaces allow to define a notion of regularity for functions.

Definition 1. A locally bounded function $f: \mathbf{R}^{n} \rightarrow \mathbf{R}$ belongs to the Hölder space $\Lambda^{\alpha}\left(x_{0}\right)$ (with $x_{0} \in \mathbf{R}^{n}$ and $\alpha \geq 0$ ) if there exist two constants $C, R>0$ and a polynomial $P$ of degree less than $\alpha$ such that

$$
\sup _{x \in B\left(x_{0}, r\right)}|f(x)-P(x)|<C\left|B\left(x_{0}, r\right)\right|^{\alpha},
$$

for any $0<r<R$. The polynomial is clearly unique and one has $\Lambda^{\alpha+\varepsilon}\left(x_{0}\right) \subset \Lambda^{\alpha}\left(x_{0}\right)$ for any $\varepsilon \geq 0$. The Hölder exponent of $f$ at $x_{0}$ is defined by

$$
h_{f}\left(x_{0}\right)=\sup \left\{\alpha \geq 0: f \in \Lambda^{\alpha}\left(x_{0}\right)\right\} .
$$

If $h_{f}$ takes only one finite value, $f$ is said to be monofractal. If $h_{f}$ takes at least two finite values, $f$ is said to be multifractal. As usual, we will write $f \in \Lambda^{\alpha}\left(\mathbf{R}^{n}\right)$ if $f \in \Lambda^{\alpha}\left(x_{0}\right)$ for any $x_{0} \in \mathbf{R}^{n}$, the constant $C$ in inequality (1) being uniform. If the Hölder exponent $h_{f}$ is not a constant function, just knowing that a function $f$ belongs to some uniform Hölder space does not give enough information about the pointwise regularity. On the other hand, the behavior of the function $h_{f}$ can be very erratic;

\footnotetext{
* Corresponding author .

E-mail addresses: tkleyntssens@ulg.ac.be (T. Kleyntssens), celine.esser@math.univ-lille1.fr (C. Esser), S.Nicolay@ulg.ac.be (S. Nicolay).
} 
in particular, obtaining $h_{f}$ from a general signal $f$ (i.e. a function obtained from real-life data) is an insuperable task from a practical point of view. Indeed, in many cases, the sets

$$
E_{h}^{f}=\left\{x \in \mathbf{R}^{n}: h_{f}(x)=h\right\}
$$

are themselves fractal sets (see [5-12]). Roughly speaking, the information about the (Hölder-)smoothness of a function is summarized by its Hölder spectrum, which we will now define.

Definition 2. The Hölder spectrum of a locally bounded function $f$ (defined on $\mathbf{R}^{n}$ ) is the function

$$
d_{f}:[0,+\infty] \rightarrow\{-\infty\} \cup[0, n] \quad h \mapsto \operatorname{dim}_{\mathcal{H}}\left(E_{h}^{f}\right),
$$

where $\operatorname{dim}_{\mathcal{H}}$ denotes the Hausdorff dimension (which is well suited for the study of multifractal sets) [13].

If $f$ is a monofractal function with a Hölder exponent equal to $H$, then $d_{f}(h)=n$ if $h=H$, and $d_{f}(h)=-\infty$ if $h \neq H$. A classical example is the Weierstraß function [14].

If $f$ is a multifractal function, the Hölder spectrum can be a concave or non-concave function. Cascades are classical examples of functions with a concave spectrum [15]. A non-concave spectrum can be obtained, for example, by summing cascades (see Theorem (1)).

Here again, since the definition of the Hölder spectrum involves successive intricate limits, there is no algorithm to directly obtain the spectrum $d_{f}$ associated with a signal $f$. However, there exist methods to estimate $d_{f}$ starting from $f$; as we will see, they only lead to upper bounds and only give the exact spectrum for specific cases.

A multifractal formalism is a method that leads to an estimation of $d_{f}$; if, when applied to a signal $f$, the procedure leads to the exact spectrum, one says that the multifractal formalism holds for $f$. Such a method was first proposed by Parisi and Frish [16]. The idea is to define the scaling function (for $q \geq 1$, provided that such an expression makes sense)

$$
S(h, q)=\int|f(t+h)-f(t)|^{q} d t
$$

(one may have to use higher order differences) to estimate the quantity

$$
\eta_{f}(q)=\liminf _{h \rightarrow 0} \frac{\log S(h, q)}{\log |h|} .
$$

Using an heuristic argument [17], one can expect to have

$$
d_{f}(h)=\inf _{q}\left\{h q-\eta_{f}(q)\right\}+n
$$

From a theoretical point of view [8,18], this method gives, at best, an upper bound. Besides, the function $\eta_{f}$ can be defined in terms of Besov spaces for $q>0$ (see [8]). Since these spaces can be characterized using wavelets, so do the function $\eta_{f}$. It leads to a very practical way for estimating the Hölder spectrum $[8,19]$. For example, such a formalism holds for the self-similar functions $[20,21]$.

Equality (2) implies that the multifractal formalism based on Besov spaces will always lead to an increasing concave estimation of the spectrum. These are indeed limitations to the range of validity of this multifractal formalism. The first one (i.e. the fact that the estimation is an increasing function) can be partially taken care of by replacing the Besov spaces $B_{q, \infty}^{S / q}$ (which are not defined for $q \leq 0$ ) with oscillation spaces [22] to obtain the so-called wavelet leaders method (WLM) [23] (see also [24] for a continuous wavelet transform approach and [10] for a comparison between the two methods); such spaces partially generalize the Besov spaces [22]. To overcome the second problem, that is to be able to recover nonconcave spectra, Jaffard introduced a new multifractal formalism, relying on the $S^{v}$ spaces [25]. In some cases (which will be explicitly defined below), such spaces can be seen as an intersection of Besov spaces [26]. Since the $S^{\nu}$ spaces are defined from the histogram of wavelet coefficients at each scale (see Definitions (3) and (4)), they are more natural from a practical point of view than the wavelet leaders used to define the oscillation spaces. For the WLM, there are simple and efficient algorithms for approximating the Hölder spectrum. For the case of the $S^{v}$ spaces, the situation is different: although there have been several attempts, a mature algorithm with a very limited number of tuning parameters is still missing.

Although $S^{v}$ spaces bring new possibilities to the multifractal analysis landscape, we will show that the transition from theory to practice is not straightforward. In this paper, we present an implementation of the formalism based on these spaces and show its effectiveness by applying it to several statistical and deterministic examples. Three theoretical results are also contained in this work. The first one (see Proposition 2) is easy to obtain and gives extra freedom for the computation of some numerical values related to the $S^{v}$ spaces. The second one (see Proposition 3 ) shows that if the signal $f$ comes from a measure (as the cascades which models the turbulence for example) the computation of the spectrum can be simplified. The third one (see Theorem 1) leads to the Hölder spectrum of the sum of two binomial cascades. We also show that the estimation of the theoretical spectrum can be obtained more easily for a large class of measure-based functions.

One could argue that such a formalism is useless, since most of the multifractal analysis previously performed led to concave spectra. Let us emphasize once more that the estimations obtained via an equality such as (2) necessary leads to a concave estimation, even if the real spectrum is not concave. It is therefore interesting to check if the previously collected estimations can be supposed concave, by comparing them to the ones obtained with the $S^{\nu}$-based multifractal formalism. 
The remainder of this paper is structured as follows. We first introduce the $S^{v}$ spaces and define the multifractal formalism they induce. Then, we show that the formalism holds for the sum of two binomial cascades. Next, we describe an algorithm based on this formalism and apply it to several examples. We start with monofractal functions and processes before shifting to the case of multifractal data. We show that in most of the cases the numerical results are better than the multifractal formalisms previously introduced and, as expected, that this approach allows to recover non-concave spectra. Finally, we look at the advantages and the drawbacks of this method. Let us remark that numerical methods based on similar ideas have been proposed [27], but they rely on parameters that have to be estimated for each considered signal.

\section{The $S^{v}$ spaces: a brief review}

The $S^{v}$ spaces were introduced in [25] and a related multifractal formalism was proposed in [28]. Concerning the validity of this formalism, it has been studied using two notions of genericity: Baire genericity and prevalence (see [29,30] for the definition of this last notion). It is not limited to concave spectra.

\subsection{The discrete wavelet transform}

Let us briefly recall some definitions and notations (for more precisions, see e.g. [31-33]). Under some general assumptions, there exist $2^{n}-1$ functions $\left(\psi^{(i)}\right)_{1 \leq i<2^{n}}$, called (periodized) wavelets, such that for any function $f \in L^{2}\left([0,1]^{n}\right)$, one has

$$
f(x)=\sum_{j \in \mathbf{N}} \sum_{k \in\left\{0, \ldots, 2^{j}-1\right\}^{n}} \sum_{1 \leq i<2^{n}} c_{j, k}^{(i)} \psi^{(i)}\left(2^{j} x-k\right),
$$

where the (periodized) wavelet coefficients are defined by

$$
c_{j, k}^{(i)}=2^{n j} \int_{[0,1]^{n}} f(x) \psi^{(i)}\left(2^{j} x-k\right) d x .
$$

Let us remark that we do not choose the $L^{2}$ normalization for the wavelets, but rather an $L^{\infty}$ normalization, which is better fitted to the study of the Hölderian regularity. Intuitively, if the point $x_{0}$ belongs to the dyadic cube $\frac{k}{2^{j}}+\left[0, \frac{1}{2^{j}}\left[^{n}\right.\right.$ then, for any $i$, one has [34]

$$
h_{f}(x)=\liminf _{j \rightarrow+\infty} \inf _{k} \frac{\log \left|c_{j, k}^{(i)}\right|}{\log \left(2^{-j}+\left|k 2^{-j}-x\right|\right)},
$$

i.e. $\left|c_{j, k}^{(i)}\right| \sim 2^{-h_{f}\left(x_{0}\right) j}$. In the sequel, we omit any reference to the index $i$. For instance, the set $\left\{c_{j, k}:(j, k) \in E\right\}$ has to be understood as the set $\left\{c_{j, k}^{(i)}:(j, k) \in E, 1 \leq i<2^{n}\right\}$.

\subsection{Definition of $\mathrm{S}^{v}$ spaces}

The main idea behind the definition of the $S^{v}$ spaces is the use of histograms of wavelet coefficients for large scales: from Eq. (3), the coefficients which behaves as $2^{-\alpha j}$ are the signature of points with a regularity $\alpha$. It seems therefore natural to try to estimate the spectrum of a function $f \in L^{2}\left([0,1]^{n}\right)$ by considering the function

$$
\rho_{f}(h)=\lim _{\varepsilon \rightarrow 0^{+}} \limsup _{j \rightarrow+\infty} \frac{\log \#\left\{k: 2^{-(h+\varepsilon) j} \leq\left|c_{j, k}\right|<2^{-(h-\varepsilon) j}\right\}}{\log 2^{j}} .
$$

There thus exist approximately $2^{\rho_{f}(h) j}$ wavelet coefficients of amplitude $2^{-h j}$. From the definition of the Hausdorff dimension and the wavelet characterization of the Hölder exponent, one can hope to have $\rho_{f}(h)=d_{f}(h)$. However, two problems make this formalism useless: the function $\rho_{f}$ depends on the chosen wavelet basis [25] and a numerical approximation of this function is difficult to obtain. Indeed, in practice, we only have a finite number of wavelet coefficients and in order to compute the double limit, one must introduce an arbitrary dependence between $\varepsilon$ and $j$ to approximate $\rho_{f}(h)$. To overcome these issues, one modifies the definition of this function to count the number of wavelet coefficients greater in modulus than $2^{-\alpha j}$ at each scale $j$. This modification allows to define a function that is independent of the wavelet basis (see below) and which can easily be computed for real-life signals (see Section 4).

Definition 3. The wavelet profile of $f \in L^{2}\left([0,1]^{n}\right)$ is defined by

$$
v_{f}(\alpha)=\lim _{\varepsilon \rightarrow 0^{+}} \limsup _{j \rightarrow+\infty} \frac{\log \# E_{j}(1, \alpha+\varepsilon)(f)}{\log 2^{j}},
$$

where

$$
E_{j}(C, \alpha)(f)=\left\{k:\left|c_{j, k}\right| \geq C 2^{-\alpha j}\right\}
$$

and $\left(c_{j, k}\right)_{j \in \mathbb{N}, k \in\left\{0, \ldots, 2^{j}-1\right\}^{n}}$ are the (periodized) wavelet coefficients of $f$. 
If $f \in \Lambda^{\varepsilon}\left(\mathbf{R}^{n}\right)$ for some $\varepsilon>0$, the function $v_{f}$ is non-decreasing, right-continuous and there exists $\alpha_{\text {min }}>0$ for which $v_{f}(\alpha)=-\infty$ for all $\alpha<\alpha_{\min }$. Moreover, one has $v_{f}(\alpha) \in[0, n]$ for all $\alpha \geq \alpha_{\min }$. A function satisfying those properties is called an admissible profile.

In this section, we follow the path laid down in [25] and [26].

Definition 4. Given an admissible profile $v: \mathbf{R} \rightarrow\{-\infty\} \cup[0, n]$, we define the space $S^{v}$ by

$$
S^{v}=\left\{f \in L^{2}\left([0,1]^{n}\right): v_{f}(\alpha) \leq v(\alpha) \forall \alpha \in \mathbf{R}\right\} .
$$

The definitions of $v_{f}$ and $S^{v}$ are independent of the chosen wavelet basis and the spaces $S^{v}$ are vector spaces linked with the Besov spaces by the following embedding:

$$
S^{\nu} \subset \bigcap_{q>0} \bigcap_{\varepsilon>0} B_{q, \infty}^{\eta(q) / q-\varepsilon},
$$

where $\eta$ is defined by

$$
\eta(q)=\inf _{\alpha \geq \alpha_{\min }}\{\alpha q-v(\alpha)\}+n
$$

The preceding inclusion becomes an equality if and only if $v$ is a concave function, in which case the corresponding space brings nothing new to the already existing formalisms. However, for non-concave profiles $v$, the contribution of the related spaces will become evident. If we denote

$$
h_{\max }=\inf _{h \geq \alpha_{\min }} \frac{h}{v(h)},
$$

the multifractal formalism based on the $S^{v}$ spaces relies on the following estimation of the Hölder spectrum:

$$
d_{f}^{v}(h)= \begin{cases}h \sup _{h^{\prime} \in(0, h]} \frac{v\left(h^{\prime}\right)}{h^{\prime}} & \text { if } h \leq h_{\max } \\ n & \text { else }\end{cases}
$$

For any $f \in S^{v}$, we have $d_{f}(h) \leq d_{f}^{v}(h)$ for all $h \geq 0$ [35] and the set

$$
\left\{f \in S^{v}: d_{f}(h)=\left\{\begin{array}{ll}
d_{f}^{v}(h) & \text { if } h \leq h_{\max } \\
-\infty & \text { else }
\end{array}\right\}\right.
$$

is generic from both the prevalence [28] and the Baire genericity [36] points of view.

\section{A theoretical example of signal with a non-concave spectrum}

In this section, we present signals with non-concave spectra for which the multifractal formalism based on the $S^{v}$ spaces holds, while the WLM (or other Legendre-based methods) only leads to an upper bound of the spectrum.

To this purpose, we consider the sum of two binomial cascades (which are the simplest deterministic case of cascades). The notion of cascade is very important since it models many phenomena, many of them occurring in turbulences [1,37].

Definition 5. The binomial cascade of parameter $p \in(0,1)$ is the only Borel measure $m$ defined on $[0,1]$ such that

$$
m\left(\left[\sum_{k=1}^{n} \frac{\varepsilon_{k}}{2^{k}}, \sum_{k=1}^{n} \frac{\varepsilon_{k}}{2^{k}}+\frac{1}{2^{n}}\right)\right)=p^{\sum_{k=1}^{n} \varepsilon_{k}}(1-p)^{n-\sum_{k=1}^{n} \varepsilon_{k}},
$$

for all $n \in \mathbf{N}$ and $\varepsilon_{k} \in\{0,1\}(k \in\{0, \ldots, n\})$.

Let us recall that if $m$ is a finite Borel measure on $[0,1]^{n}$, the definition of Hölder exponent of $x_{0}$ and the Hölder spectrum of $m$ are respectively [38]

$$
h_{m}\left(x_{0}\right)=\liminf _{r \rightarrow 0^{+}} \frac{\log m(B(x, r))}{\log r}
$$

and

$$
d_{m}:[0,+\infty] \rightarrow\{-\infty\} \cup[0, n] \quad h \mapsto \operatorname{dim}_{\mathcal{H}}\left(\left\{x \in \mathbf{R}^{n}: h_{m}(x)=h\right\}\right) .
$$

If $m$ is an uniformly regular measure (i.e. there exist a constant $C>0$ and an exponent $h_{\min }>0$ such that $m(B(x, r)) \leq C r^{h_{\min }}$ for any ball $\left.B(x, r) \subset[0,1]^{n}\right)$ then there exist a function $f$, called the associated wavelet series, whose wavelet coefficients are defined by $c_{j, k}=m\left(\left[k 2^{-j},(k+1) 2^{-j}\right)\right)$. In this case, $d_{f}=d_{m}$ (for more details, we refer the reader to [38,39]). As we will see in Proposition 3 (see Section 4), in this setting, the $S^{v}$-based method reduces to the computation of the wavelet profile $v_{f}$ (so that we can avoid the estimation of $d_{f}^{v_{f}}$ ). 
The Hölder spectrum of a binomial cascade of parameter $p<1 / 2$ is

$$
d_{m}(h)=-\left(\alpha \log _{2}(\alpha)+(1-\alpha) \log _{2}(1-\alpha)\right),
$$

where

$$
\alpha=\frac{h+\log _{2}(1-p)}{\log _{2}(1-p)-\log _{2} p},
$$

for all $h \in\left[-\log _{2}(1-p),-\log _{2} p\right]$ (for a proof, see e.g. [15]).

The following theorem gives the Hölder spectrum of the sum of two binomial cascades.

Theorem 1. Let us denote by $m_{p_{1}}$ (resp. $m_{p_{2}}$ ) the binomial cascade of parameter $p_{1}$ (resp. $p_{2}$ ), with $0<p_{1}<p_{2}<1 / 2$. The Hölder spectrum of the measure $m_{p_{1}}+m_{p_{2}}$ is equal to

$$
d_{m_{p_{1}}+m_{p_{2}}}(h)=\left\{\begin{array}{lll}
d_{m_{p_{1}}}(h) & \text { if } & h \leq h_{0} \\
d_{m_{p_{2}}}(h) & \text { if } \quad h \geq h_{0}
\end{array},\right.
$$

for all $h \in\left[-\log _{2}\left(1-p_{1}\right),-\log _{2} p_{2}\right]$, where

$$
h_{0}=\frac{\log _{2}\left(1-p_{1}\right) \log _{2} p_{2}-\log _{2}\left(1-p_{2}\right) \log _{2} p_{1}}{\log _{2}\left(\frac{1-p_{2}}{p_{2}}\right)-\log _{2}\left(\frac{1-p_{1}}{p_{1}}\right)} .
$$

Proof. Let us first remark that for any $x \in[0,1]$,

$$
h_{m_{p_{1}}+m_{p_{2}}}(x)=\min \left\{h_{m_{p_{1}}}(x), h_{m_{p_{2}}}(x)\right\} .
$$

Indeed, for every $\varepsilon>0$, there is $0<R<1$ such that

$$
m_{p_{1}}(B(x, r)) \leq r^{h_{m_{1}}}(x)-\varepsilon \text { and } \mu_{p_{2}}(B(x, r)) \leq r^{h_{m_{p_{2}}}(x)-\varepsilon}
$$

for every $r<R$. One has

$$
\begin{aligned}
\frac{\log \left(m_{p_{1}}(B(x, r))+m_{p_{2}}(B(x, r))\right)}{\log r} & \geq \frac{\log \left(r^{h_{m_{p_{1}}}(x)-\varepsilon}+r^{h_{m_{p_{2}}}(x)-\varepsilon}\right)}{\log r} \\
& \geq \frac{\log \left(2 r^{\min \left\{h_{m_{p_{1}}}(x), h_{m_{p_{2}}}(x)\right\}-\varepsilon}\right)}{\log r},
\end{aligned}
$$

for every $r<R$. It follows that $h_{m_{p_{1}}+m_{p_{2}}}(x) \geq \min \left\{h_{m_{p_{1}}}(x), h_{m_{p_{2}}}(x)\right\}-\varepsilon$ and since $\varepsilon>0$ is arbitrary, we get that $h_{m_{p_{1}}+m_{p_{2}}}(x) \geq \min \left\{h_{m_{p_{1}}}(x), h_{m_{p_{2}}}(x)\right\}$.

In view of

$$
m_{p_{1}}(B(x, r))+m_{p_{2}}(B(x, r)) \geq m_{p_{1}}(B(x, r))
$$

and

$$
m_{p_{1}}(B(x, r))+m_{p_{2}}(B(x, r)) \geq m_{p_{2}}(B(x, r)),
$$

the other inequality is obvious.

For any $x \in[0,1]$, let $S_{n}(x)$ denote the number of 0 's appearing in the $n$th first terms of the proper dyadic development of $x$. If $m_{p}$ is the binomial cascade of parameter $p<1 / 2$, it is easy to check that

$$
\liminf _{n \rightarrow+\infty} \frac{S_{n}(x)}{n}=\frac{h_{m_{p}}(x)+\log _{2}(1-p)}{\log _{2}(1-p)-\log _{2} p} .
$$

Consequently, for every $x \in[0,1]$, we have

$$
\frac{h_{m_{p_{1}}}(x)+\log _{2}\left(1-p_{1}\right)}{\log _{2}\left(1-p_{1}\right)-\log _{2} p_{1}}=\frac{h_{m_{p_{2}}}(x)+\log _{2}\left(1-p_{2}\right)}{\log _{2}\left(1-p_{2}\right)-\log _{2} p_{2}}
$$

and a simple computation shows that

$$
h_{m_{p_{1}}+m_{p_{2}}}(x)=h_{m_{2}}(x) \Longleftrightarrow h_{m_{2}}(x) \geq h_{0} .
$$

The conclusion follows.

Remark 1. Let us notice that $d_{m_{p_{1}}}\left(h_{0}\right)=d_{m_{p_{2}}}\left(h_{0}\right)$ and that $h_{0}$ corresponds to the first intersection of the two graphs since we have

$$
h_{0}<\frac{-\log _{2}\left(1-p_{2}\right)-\log _{2} p_{2}}{2} .
$$




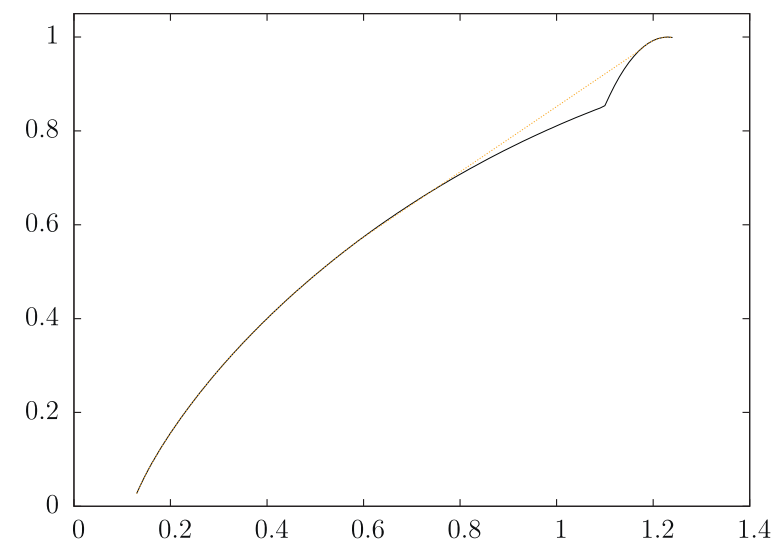

Fig. 1. Spectrum of the sum of two binomial cascades of parameters $p_{1}=0.08$ and $p_{2}=0.4$ where the second cascade is shifted to the right by 0.2 . Theoretical spectrum (-) and WLM spectrum (_- ). The spectrum obtained with the $S^{\nu}$ spaces corresponds to the theoretical spectrum.

Remark 2. This last result can easily be generalized to the case where a binomial cascade $m$ is shifted by a parameter $\beta>0$, that is to say that the wavelet coefficients of the associated wavelet series are given by $2^{-\beta j} m\left(\left[k 2^{-j},(k+1) 2^{-j}\right)\right)$ for every $j \in \mathbf{N}, k \in\left\{0, \ldots, 2^{j}-1\right\}$. In this case, we get that the Hölder spectrum of the sum of a binomial cascade $m_{1}$ of parameter $p_{1}$ and a shifted binomial cascade $m_{2}$ of parameter $p_{2}$ (with $0<p_{1}<p_{2}<1 / 2$ ) with shifting parameter $\beta$ is given by

$$
d_{m_{1}+m_{2}}(h)=\left\{\begin{array}{lll}
d_{m_{1}}(h) & \text { if } & h \leq h_{0} \\
d_{m_{2}}(h) & \text { if } & h \geq h_{0}
\end{array}\right.
$$

for any $h \in\left[-\log _{2}\left(1-p_{1}\right), \min \left\{-\log _{2} p_{1},-\log _{2} p_{2}+\beta\right\}\right]$, where $h_{0}$ is the first intersection of the graphs of $d_{m_{1}}$ and $d_{m_{2}}$. Fig. 1 shows an example.

One easily checks that the spectrum obtained with the $S^{v}$ spaces is exactly the theoretical spectrum. Indeed, a classical result of the theory of large deviations states that the increasing part of the spectrum of a binomial cascade is equal to its wavelet profile (see e.g. [40]). Since $S^{v}$ spaces are vector spaces, the wavelet profile of the sum is smaller than the Hölder spectrum. The other inequality is due to Proposition 3, proved in Section 4. Obviously, on the opposite, the WLM only gives the concave hull of the spectrum.

\section{Implementation of the multifractal formalism based on the $S^{v}$ spaces}

Now that the theoretical setting has been formulated and the theoretical interest has been shown, we can describe our algorithm to effectively compute the spectrum $d_{f}^{v}$ associated with a numerical signal $f$ : first, we show that $v_{f}$ can be theoretically replaced by the function $v_{f}^{C}$ defined by

$$
v_{f}^{C}(\alpha)=\lim _{\varepsilon \rightarrow 0^{+}} \limsup _{j \rightarrow+\infty} \frac{\log \# E_{j}(C, \alpha+\varepsilon)(f)}{\log 2^{j}},
$$

for any $C>0$. Next, we explain how to compute the function $v_{f}^{C}$ and give a way to effectively choose the constant $C$. Let us mention that the algorithm we propose is a stand-alone method: no operation but fine tuning is required by the user.

Of course, in practice, a signal is a finite sequence of values. We use the Mallat algorithm [41] and the Daubechies wavelets [42] to compute a finite number of wavelet coefficients. In $\mathbf{R}^{n}$ we adopt the tensor product wavelet basis [31,32].

\subsection{A preliminary result}

The following proposition is the key result for the effective implementation of the formalism.

Proposition 2. Let $\mathbf{C}=\left(C_{j}\right)_{j \in \mathbf{N}}$ be a sequence of positive numbers such that there exists $c>0$ such that $1 / c \leq C_{j} \leq c$ for any $j \in \mathbf{N}$. If we denote

$$
v_{f}^{C}(\alpha)=\lim _{\varepsilon \rightarrow 0^{+}} \limsup _{j \rightarrow+\infty} \frac{\log \# E_{j}\left(C_{j}, \alpha+\varepsilon\right)(f)}{\log 2^{j}},
$$

we have $v_{f}^{\mathbf{C}}(\alpha)=v_{f}(\alpha)$ for any $\alpha \in \mathbf{R}$.

If $\boldsymbol{C}$ is the constant sequence $C_{j}=C$, we naturally write $v_{f}^{C}$ by $v_{f}^{C}$. 
Proof. First, observe that

$$
\# E_{j}(1 / c, \alpha+\varepsilon) \leq \# E_{j}\left(C_{j}, \alpha+\varepsilon\right) \leq \# E_{j}(c, \alpha+\varepsilon),
$$

for all $j \in \mathbf{N}, \alpha \in \mathbf{R}$ and $\varepsilon>0$. So that we have $v_{f}^{1 / c} \leq v_{f}^{C} \leq v_{f}^{c}$; therefore it is enough to prove that we have $v_{f}^{C}=v_{f}$ for any $C>0$.

Fix $\alpha \in \mathbf{R}, C>0, \varepsilon>0$ and let $J>0$ be such that for all $j>J, C 2^{-\varepsilon / 2 j}<1$. If $\left|c_{j, k}\right| \geq 2^{-(\alpha+\varepsilon / 2) j}$ then

$$
\left|c_{j, k}\right| \geq 2^{-(\alpha+\varepsilon / 2) j} C 2^{-\varepsilon / 2 j}=C 2^{-(\alpha+\varepsilon) j}
$$

in other words, we have

$$
\# E_{j}(C, \alpha+\varepsilon)(f) \geq \# E_{j}(1, \alpha+\varepsilon / 2)(f),
$$

which implies $v_{f}^{C}(\alpha) \geq v_{f}(\alpha)$.

For the other inequality, it suffices to take $J>0$ such that $2^{-\varepsilon j}<C$ for all $j>J$.

\subsection{Computation of $\nu_{f}^{C}(\alpha)$ for fixed $\mathrm{C}$ and $\alpha$}

Let us recall that the definition of $\nu_{f}^{C}(\alpha)$ formalizes the idea that, if $\alpha \geq \alpha_{\min }$, there are about $2^{v_{f}^{C}(\alpha) j}$ coefficients larger than $C 2^{-\alpha j}$ at scales $j$ "large enough". Following this idea, an approximation of $\nu_{f}^{C}(\alpha)$ is given by the slope of

$$
j \mapsto \frac{\log \# E_{j}(C, \alpha)(f)}{\log 2},
$$

for $j$ "large enough". In practice, it is approximated by the slope of a linear regression on correlated points only, using the least squares method. Also, a lower bound for the correlation has to be fixed. If the correlation is always smaller that this lower bound or if $\# E_{j}(C, \alpha)(f)=0$ for several $j$ 's, we say that the detected value for $v_{f}^{C}(\alpha)$ is $-\infty$. The impact of the choice of this lower bound (the typical values used are $p=0.95,0.99,0.999)$ will be illustrated in the next section with examples. From now on, the notation $v_{f}^{C}(\alpha)$ will refer to this slope.

\subsection{For a fixed $\alpha>0$, choice of the constant $\mathrm{C}>0$}

From a theoretical point of view, the spectrum $d_{f}^{v_{f}}$ can be estimated by the computation of $v_{f}$, as introduced in Definition 3. However in practice, since we only have access to a finite number of wavelet coefficients, such a method is not efficient. If the typical value of these coefficients is too big (resp. too small) with respect to 1 , too many (resp. not enough) of them will be taken into account, so that the associated value of $v_{f}(\alpha)$ will be 1 (resp. $\left.-\infty\right)$.

In order to determine a good approximation of $v_{f}(\alpha)$, we use Proposition 2, that allows us to replace the constant 1 by an arbitrary constant $C$, which has to be adapted to the amplitude of the wavelet coefficients. Consequently, the main problem, for a fixed $\alpha>0$, lies in the choice of the constant $C$. If $\alpha \geq \alpha_{\min }$, it should exist an interval $\left[C_{1}, C_{2}\right]$ such that for any $C \in\left[C_{1}\right.$, $C_{2}$ ], the values $v_{f}^{C}(\alpha)$ are close. For every fixed $\alpha>0$, we construct the function $C>0 \mapsto v_{f}^{C}(\alpha)$.

If $\alpha \geq \alpha_{\min }$, this function should be decreasing and a stabilization should appear. In the other case (i.e. if $\alpha<\alpha$ min), the function should decrease without stabilization. We compute this function starting with $C=0$ until the detected value of $v_{f}^{C}(\alpha)$ is $-\infty$.

To detect the existence of a stabilization, we use a non-parametric gradient descent, where the gradient is calculated as the slope of the regression line over several consecutive points (typically 3 or 5 points) [43]. The approximation of $v_{f}(\alpha)$ is

- $-\infty$ if no stabilization is detected;

- the mean of the values $v_{f}^{C}(\alpha)$ obtained for the C's whose gradient are close to 0 , if a stabilization is detected.

A last crucial point is to determine the minimal length of the interval $\left[C_{1}, C_{2}\right]$ where the gradients are close to 0 . A first idea is to consider a length equal to the greatest value among the moduli of the wavelet coefficients. It gives some good results (see [44] for a similar method) but it is less efficient for signals with small size and it may be influenced by aberrant values in the signal (see Appendix). Besides, for any $\alpha$, this approach uses the same magnitude for the stabilization. However, the number of wavelet coefficients used to approximate $\nu(\alpha)$ strongly depends on $\alpha$; so does the length of the interval with a stabilization. In this article, a more robust approach is proposed for which the length of the interval [ $\left.C_{1}, C_{2}\right]$ depends on $\alpha$. Let us recall that we count the number of wavelet coefficients that are greater than $C 2^{-\alpha j}$. In other words, the considered wavelet coefficients $c_{j, k}$ satisfy $2^{\alpha j}\left|c_{j, k}\right| \geq C$. In view of this inequality, the idea is to consider an interval [ $C_{1}$, $C_{2}$ ] of magnitude equals the median of the values $2^{\alpha j}\left|c_{j, k}\right|$. In Sections 5 and 6 , we show that this method gives very good results. It is not influenced by aberrant values in the signal and allows to have a better approximation of the spectrum than other wavelet-methods. 


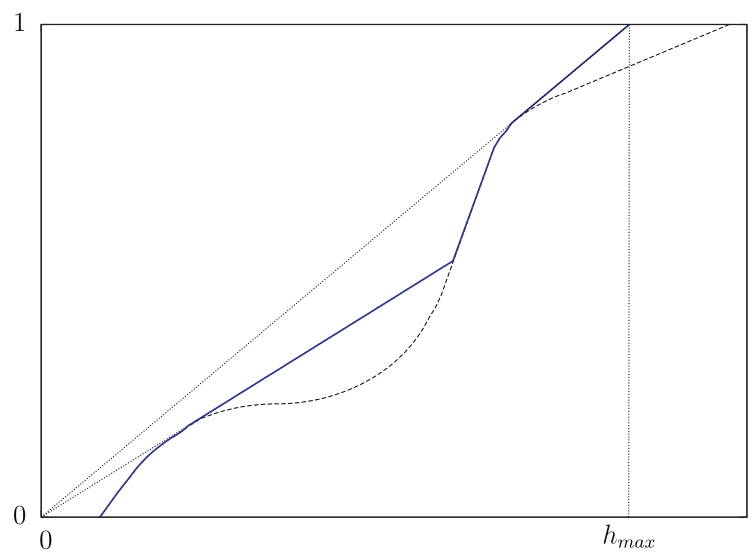

Fig. 2. Example of $v_{f}(---)$ and $d_{f}^{v_{f}}(-)$.

4.4. The transition from $v_{\mathrm{f}}$ to $d_{f}^{v_{f}}$

Let us recall that the multifractal formalism based on the $S^{v}$ spaces for a function $f$ is a transformation of the function $v_{f}$ (see Eq. (4)). To understand this transformation, let us introduce the following definition [45].

Definition 6. Let $0 \leq a<b \leq+\infty$. A function $f:[a, b] \mapsto \mathbf{R}^{+}$is with increasing-visibility if $f$ is continuous at $a$ and

$$
\sup _{y \in(a, x]} \frac{f(y)}{y} \leq \frac{f(x)}{x},
$$

for all $x \in(a, b]$.

In other words, a function $f$ is with increasing-visibility if for all $x \in(a, b]$, the segment $((0,0),(x, f(x))]$ lies above the graph of $f$ on $(a, x]$.

The passage from $v_{f}$ to $d_{f}^{v_{f}}$ transforms the function $v_{f}$ into a function with increasing-visibility. This is illustrated in Fig. 2.

In practice, once $v_{f}$ has been computed, a first natural idea is to estimate $\sup _{h^{\prime} \in(0, h]} v_{f}\left(h^{\prime}\right) / h^{\prime}$ in order to determine $d_{f}(h)$. However, since we have to deal with numerical data, there are precision errors in the function $v_{f}$ which may introduce a bias in the computation of this upper bound. In order to overcome this issue, we can use the fact that the function $v_{f}$ is transformed into a function with increasing-visibility. It is easy to see that a function $g$ is with increasing-visibility if and only if the function

$$
h>0 \mapsto \frac{g(h)}{h}
$$

is increasing. So, we can compute the function $h>0 \mapsto \frac{v_{f}(h)}{h}$ (for example, by taking steps of 0.01 ). If it is increasing, then $d_{f}(h)=v_{f}(h)$; on the other hand, if it is decreasing, we are in a non increasing-visibility part of $v_{f}$. In this case, we compute the mean of $v_{f}(h) / h$ for the first points of this decreasing part. If we denote by $s$ this mean, we use the estimation $d_{f}^{v_{f}}(h)=h s$ for every $h$ such that $v_{f}(h) / h<s$. Once we get $v_{f}(h) / h \geq s$, we study, as previously, the monotonicity of the function $h \mapsto v_{f}(h) / h$ to determine $d_{f}^{v_{f}}$.

This last step of the algorithm adds a lot of computational operations to the method. Let us show that in some cases, this step is not useful.

Proposition 3. Let $m$ be an uniformly regular measure and let $f$ be the associated wavelet series. If

$$
\inf \left\{\frac{v_{f}(x)-v_{f}(y)}{x-y}: x, y \in\left[\alpha_{\min }, \alpha_{\max }\right], x<y\right\}>0,
$$

where $\alpha_{\max }=\inf \left\{\alpha: v_{f}(\alpha)=1\right\}$, then the wavelet profile $v_{f}$ is an approximation of the increasing part of $d_{f}$, i.e. it gives an upper bound of the Hölder spectrum.

Proof. Let us remark that if $f_{\beta}$ denotes the function whose wavelet coefficients are given by $2^{-\beta j} c_{j, k}$, then we have

$$
d_{f_{\beta}}(h)=d_{f}(h-\beta) \quad \text { and } \quad v_{f_{\beta}}(h)=v_{f}(h-\beta)
$$




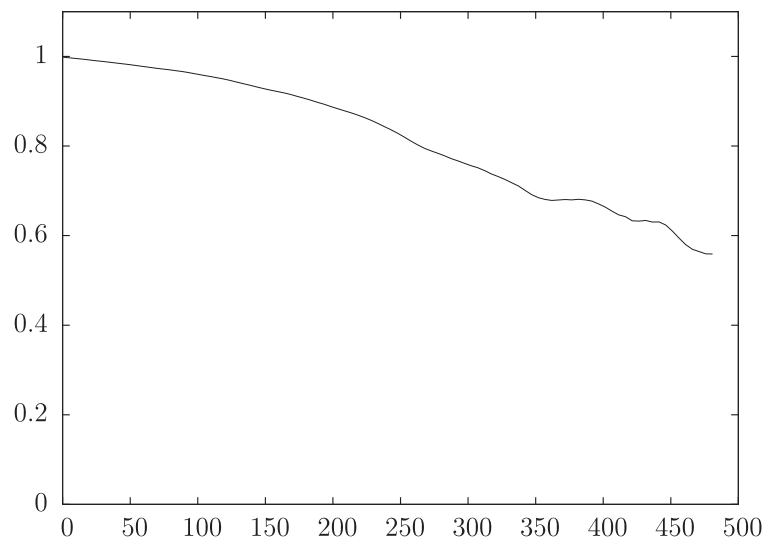

(a) $\alpha=0.45$ and $l=99.1616$

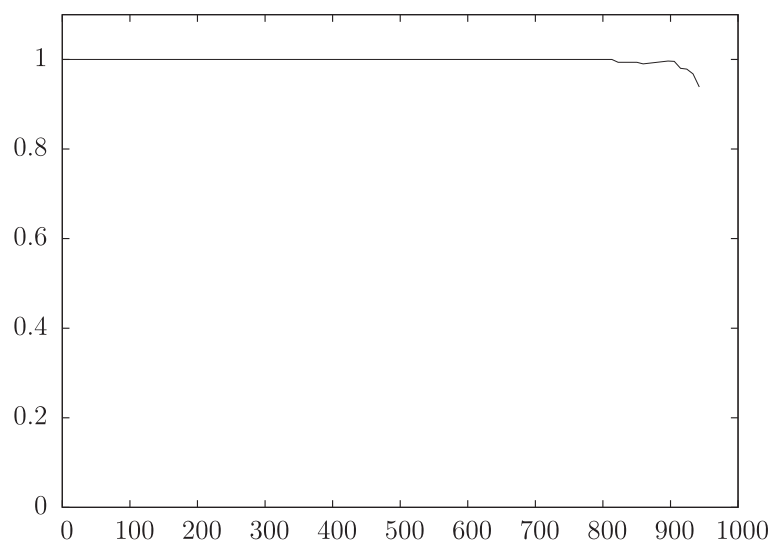

(b) $\alpha=0.5$ and $l=184.362$

Fig. 3. Function $C \mapsto \nu^{C}(\alpha)$ for a simulation of a Brownian motion. We search a stabilization on an interval [ $C_{1}$, $\left.C_{2}\right]$ whose length $l$ depends on $\alpha$ (see Section 4.3).

for any $h \in[0,+\infty]$. The hypothesis on the wavelet profile $v_{f}$ implies that there exists $\beta>0$ such that the function $v_{f_{\beta}}$ is with increasing-visibility. In this case, $v_{f_{\beta}}$ approximates $d_{f_{\beta}}$ (see the end of Section 2). Using property (6), we can conclude that the increasing part of $d_{f}$ can be approximated by $v_{f}$.

Remark 3. Obviously, if the infimum in (5) equals 0 and if the infimum taken on $x, y \in\left[\alpha_{\min }, \alpha\right]$ is strictly positive for any $\alpha<\alpha_{\max }$, then the result is still valid.

The method presented in this section will be called the Wavelet Profile Method (WPM). In the following, we will show that this method gives better theoretical results on some signals: we will do a systematic study on signal simulations by discussing the size of the signals generated and by comparing the WPM with the WLM.

\section{WPM in action: standard setting}

The next step is to test the algorithm described in Section 4 on several examples. We show here that the method gives results at least as good as results obtained using other wavelet-methods (mainly, the WLM) when the function is monofractal or multifractal with a concave spectrum.

\subsection{The fractional Brownian motion}

To illustrate the monofractal case, the walks of a fractional Brownian motion are used. This process was introduced by Mandelbrot and Van Ness in [46]. For a given $H \in[0,1]$, it is the unique Gaussian stochastic process that is self-similar of order $H$ with stationary increments. Its main property is the existence of a long-range correlation which introduces a weak dependence between the points of a realization. Such dependence are detected in many experimental observations and that is why fractional Brownian motions model many monofractal phenomena [46-49].

The parameter of self-similarity $H$ is called the Hurst index. Almost surely, the walk of a fractional Brownian motion of parameter $H \in(0,1)$ is continuous, nowhere differentiable and the associated Hölder exponent is equal to $H$ [50].

To simulate fractional Brownian motions, we use the algorithm described in [51]. As a first illustration, we compute the spectrum of a classical Brownian motion (i.e. $H=1 / 2$ ). Let us recall that to approximate the spectrum at point $\alpha$, we look for a stabilization of the function $C_{\mapsto} v^{C}(\alpha)$. If there is no stabilization, the spectrum at point $\alpha$ is equal to $-\infty$. Consequently, for a Brownian motion, no stabilization should be detected for $\alpha<0.5$ and a stabilization equal to 1 should appear for $\alpha=0.5$. This is illustrated in Fig. 3 .

For a fixed size $2^{j}(12 \leq j \leq 20)$, we have simulated one hundred walks of fractional Brownian motion with a parameter $H$ which varies between 0.2 and 0.8 by steps of 0.05 .

We have compared our method with the WLM: in Fig. 4, the mean and the boxplot of the distances between the detected Hölder exponent and $H$ for simulations of size $2^{j}$ is represented for any $j$.

The two methods often give similar results. The WPM seems more robust, in the sense that the boxplots associated with this method are smaller than those associated with the WLM. The robustness will be more deeply studied in Section 6.3. 


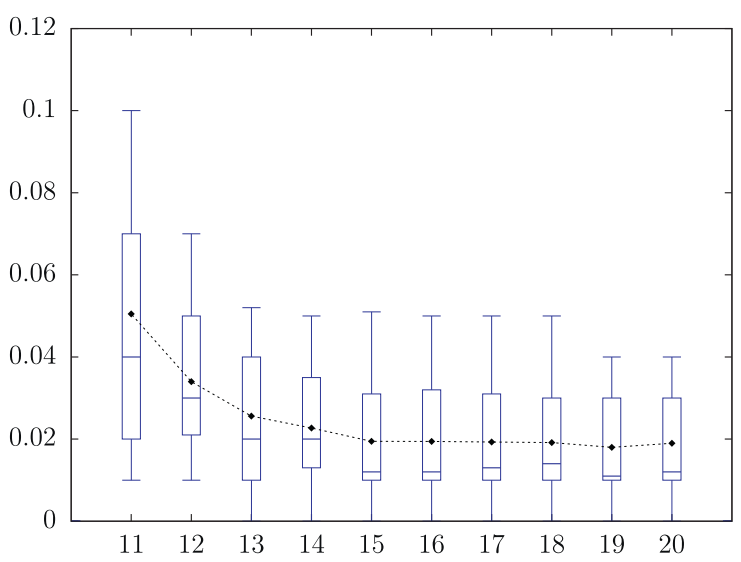

(a) WPM

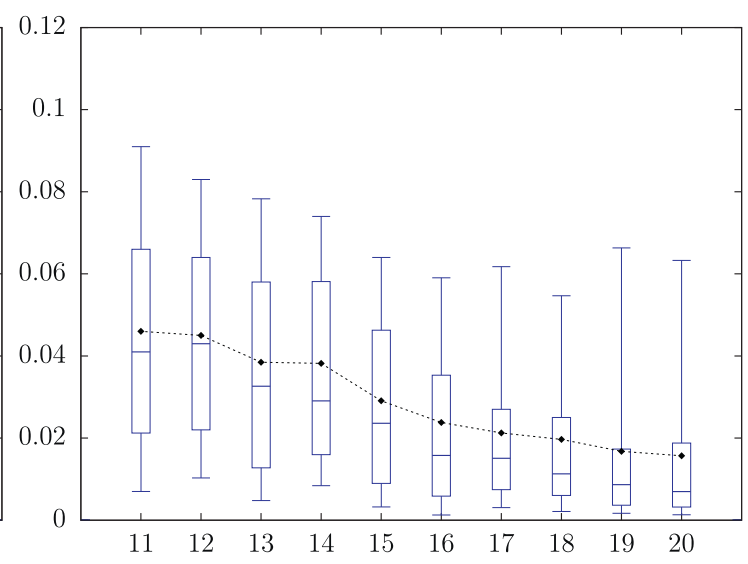

(b) WLM

Fig. 4. Functions that represent for every $j$, the boxplot of the distances between the Hölder exponent detected and $H$ (for $H$ which varies between 0.2 and 0.8 by steps of 0.05 ) of size $2^{j}$ of walks of fractional Brownian motion with a parameter $H$. The mean of these distances is also represented (in black).

\subsection{Multifractal examples with concave spectra: the Mandelbrot cascades}

The first example of function with a concave spectrum is based on the notion of Mandelbrot cascades [52]. It generalizes the notion of binomial cascades presented in Section 3.

Definition 7. Let $W$ be a given positive random variable such that $E[W]=1$ and $\left\{W_{\varepsilon}\right\}_{\varepsilon \in \Sigma}$ be an i.i.d. collection of copies of $W$ indexed with the infinite binary tree $\Sigma=\bigcup_{n \geq 1}\{0,1\}^{n}$. For any $n \in \mathbf{N}$, one defines the random measure $m_{n}$ on $[0,1]$ by

$$
m_{n}\left(\left[\sum_{k=1}^{n} \frac{\varepsilon_{k}}{2^{k}}, \sum_{k=1}^{n} \frac{\varepsilon_{k}}{2^{k}}+\frac{1}{2^{n}}\right)\right)=W_{\varepsilon_{1}} W_{\varepsilon_{1} \varepsilon_{2}}, \ldots, W_{\varepsilon_{1} \varepsilon_{2}, \ldots, \varepsilon_{n}} .
$$

The sequence $m_{n}$ converges almost surely to a Borel measure $m$ on the interval $[0,1][38]$.

In the sequel, we will consider the case where the law of $W$ is log-normal with parameters $\mu<0$ and $\sigma^{2}$ such that $|\mu| / \sigma>\sqrt{2 \log 2}$. Its spectrum is given almost surely by

$$
d(h)=-\frac{(h+\mu / \log 2)^{2} \log 2}{2 \sigma^{2}}+1
$$

if $h \in\left[-\sqrt{\frac{2}{\log 2}} \sigma-\frac{\mu}{\log 2}, \sqrt{\frac{2}{\log 2}} \sigma-\frac{\mu}{\log 2}\right][38,53]$.

The theoretical spectrum is compared with the spectrum obtained with the WPM and with the WLM in Fig. 5: one can see that both methods give a good approximation of the spectrum. Let us remark that the WPM hardly detect the spectrum for small $h$; this problem depends on the size of the signal (on average, the first $h$ is equal to 0.03 for size $2^{20}$ and 0.08 for size $2^{15}$ ). Let us recall that this method is based on the investigation of the behavior of the number of wavelet coefficients that are larger than $2^{-h j}$ across scales $j$. For very small $h$, there are not enough remaining coefficients to have a stabilization. Let us mention that the method proposed in this article (i.e. which consists in taking the median of the values $2^{\alpha j}\left|c_{j, k}\right|$ as magnitude of the size of the interval of the stabilization) greatly improves this detection compared to similar methods [44], as developed in Appendix.

For a fixed size $2^{j}(11 \leq j \leq 20)$, we have simulated one hundred log-normal cascades and computed the root-mean-square deviation (RMSE) of each simulation. The boxplot and the mean of these RMSE are represented in Fig. 6 . The two methods give very similar results.

\subsection{Multifractal examples with concave spectra: the Lévy processes without Brownian part}

The second example considered here is a Lévy process. It is a stochastic process with independent and stationary increments that is right-continuous and admits almost surely a left limit at any point. A Lévy process is associated with an index $\beta \in[0,2]$, called the Blumenthal and Getoor lower index. It is mostly used in the field of financial modeling [54].

The multifractal nature of a Lévy process is presented in [55]. Let us recall one of its main properties: a Lévy process can be decomposed into the sum of a (possibly vanishing) Brownian part and an independent pure jump process. The 


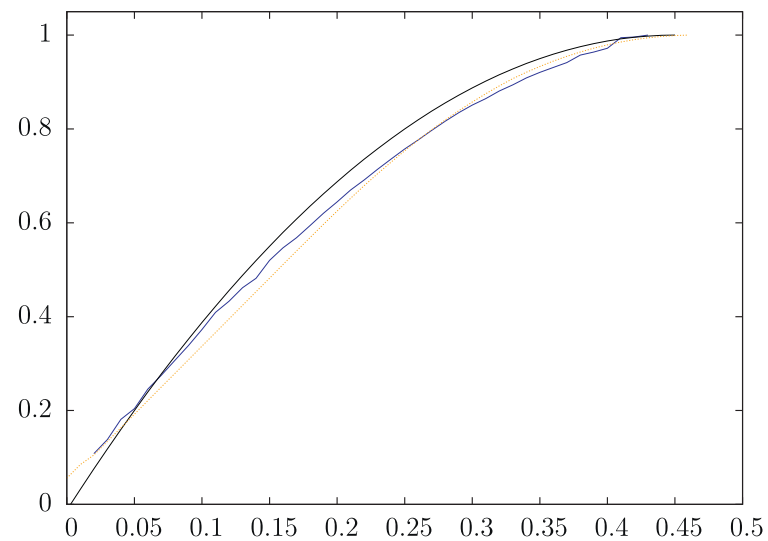

(a) size $2^{20}$

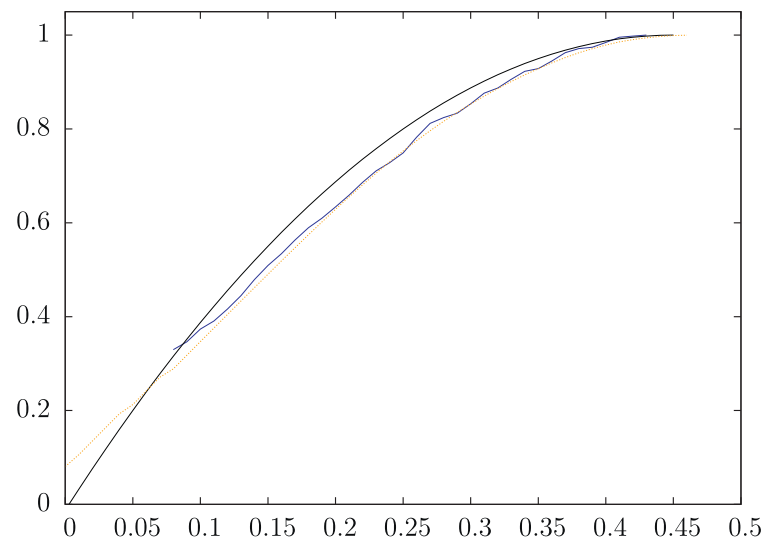

(b) size $2^{15}$

Fig. 5. Increasing part of the spectrum of the $\log$-normal cascade $\left(\mu=-0.45 \log 2\right.$ and $\left.\sigma^{2}=0.1 \log 2\right)$. Theoretical spectrum $(-)$, WPM spectrum ( - ) and WLM spectrum (

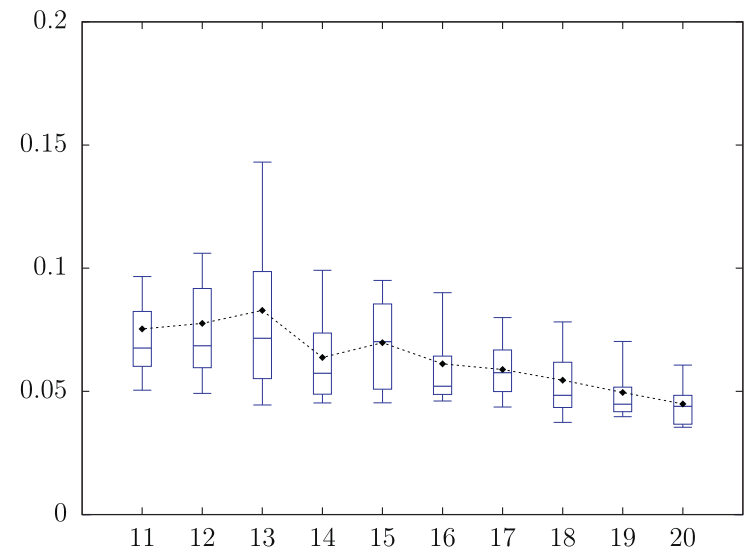

(a) WPM

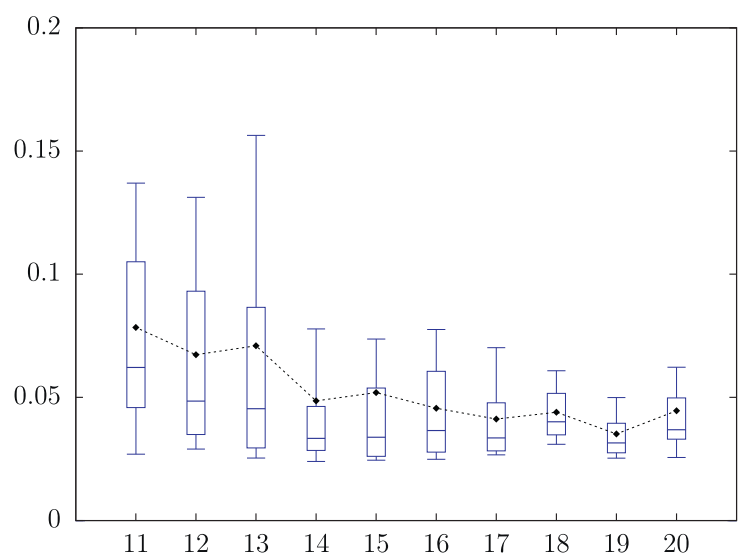

(b) WLM

Fig. 6. Functions that represent for every $j$, the boxplot of the RMSE of one hundred log-normal cascades of size $2^{j}$. The mean of these RMSE is also represented (in black).

multifractal properties of the sample paths of a Lévy process are governed by its index $\beta$ : if the Lévy process with index $\beta$ has no Brownian part, then almost surely, its spectrum is given by

$$
d(h)=\beta h
$$

for all $h \in[0,1 / \beta]$. The case where there is a non-vanishing Brownian part will be treated in the next section.

To simulate Lévy processes, we use the algorithm described in [56].

The theoretical spectrum is compared with the spectrum obtained with the WPM and with the WLM in Fig. 7. The spectrum detected with the WPM fits better the real one than the spectrum obtained via the WPM. This last method tends to make a strictly concave spectrum. However, as for the previous example, the WPM does not detect the spectrum for very small $h$ but the method proposed in this article greatly improve this detection compared to similar methods [44], as illustrated in Appendix.

For a fixed size $2^{j}(11 \leq j \leq 20)$, we have simulated one hundred Lévy processes without Brownian part $(\beta=1.3)$ and the RMSE of each simulation has been computed. The boxplot and the mean of these RMSE are represented in Fig. 8. The WPM clearly gives better results than the WLM.

\section{WPM in action: effectiveness of the method on classical examples}

In this section, we show the contributions of the WPM in the computation of the Hölder spectrum. First, we consider functions that have a non-concave spectra and we show that the WPM gives a good approximation of the spectrum. As 


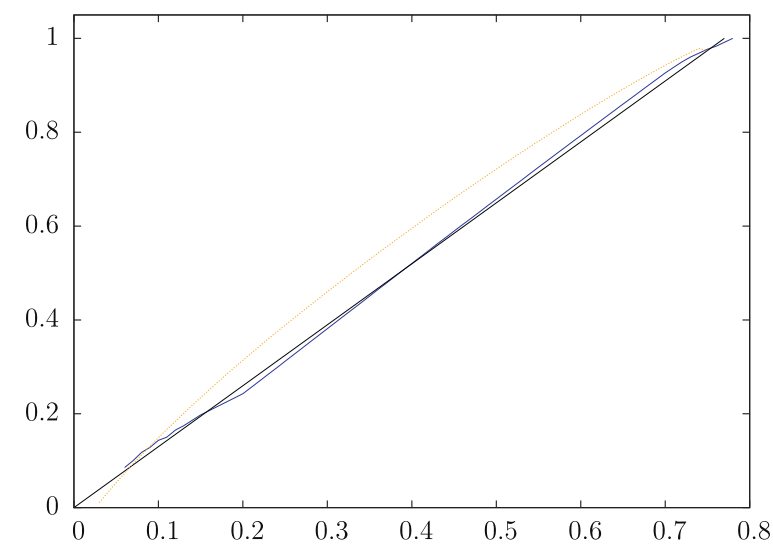

Fig. 7. Spectrum of the Lévy process without Brownian part $(\beta=1.3)$. Theoretical spectrum $(-), S^{\nu}$ spectrum ( $\_$) and WLM spectrum ( --$)$. The results are obtained with a realization of length $2^{20}$.

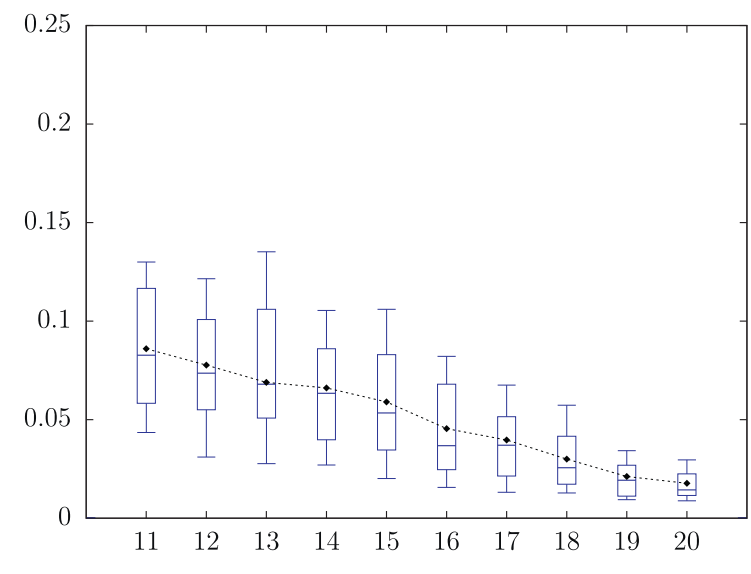

(a) WPM

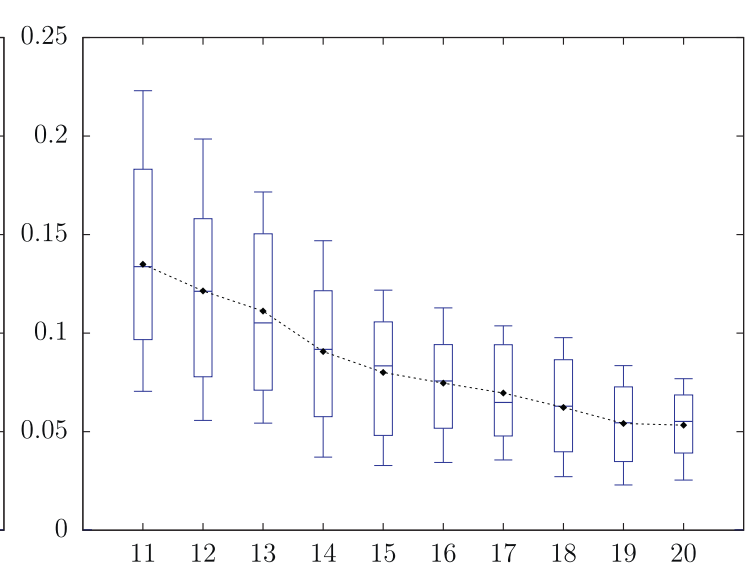

(b) WLM

Fig. 8. Functions that represent for every $j$, the boxplot of the RMSE of one hundred Lévy processes without Brownian part $\left(\beta=1.3\right.$ ) of size $2^{j}$. The mean of these RMSE is also represented (in black).

expected, the WLM only allows at best to recover the concave hull of the spectrum. Second, we illustrate that the WPM is more robust than the WLM.

\subsection{Multifractal examples with non-concave spectra: the Lévy processes with a Brownian part}

The first example with a non-concave spectrum gives a complement to the computation of the spectrum of a Lévy process. Indeed, in Section 5.3, we have calculated the Hölder spectrum of a Lévy process without Brownian part. Now, we treat the case where the Brownian motion of the Lévy process is non-vanishing [55]. If the Lévy process with index $\beta$ has a Brownian component, the associated Hölder spectrum is non-concave and, almost surely, is equal to

$$
d(h)= \begin{cases}\beta h & \text { if } h \in[0,1 / 2) \\ 1 & \text { if } h=1 / 2\end{cases}
$$

In Fig. 10, we have represented the function $C \mapsto \nu^{C}(\alpha)(\alpha=0.4$ and $\alpha=0.52)$ for a simulation of a Lévy process with a Brownian part. It is interesting to note that for $\alpha<0.5$, one stabilization is detected and corresponds to the approximation of the spectrum at $\alpha$ (see Fig. 10(a)); for $\alpha>0.5$, two stabilizations are detected (see Fig. 10(b)). This first one is the approximation of the spectrum at $\alpha$ and the second one corresponds to the Lévy process without Brownian part used to define the signal. The WPM can thus detect the presence of several processes in a single signal.

In Fig. 9, we compare the spectra obtained with the WPM and the WLM. As for the Lévy process without Brownian part, we have used the function $v_{f}$ to approximate the spectrum. With the WPM, the non-concave part is detected. In this case, the superiority of the WPM is clear, since the WLM only allows to detect the concave hull of the spectrum. 


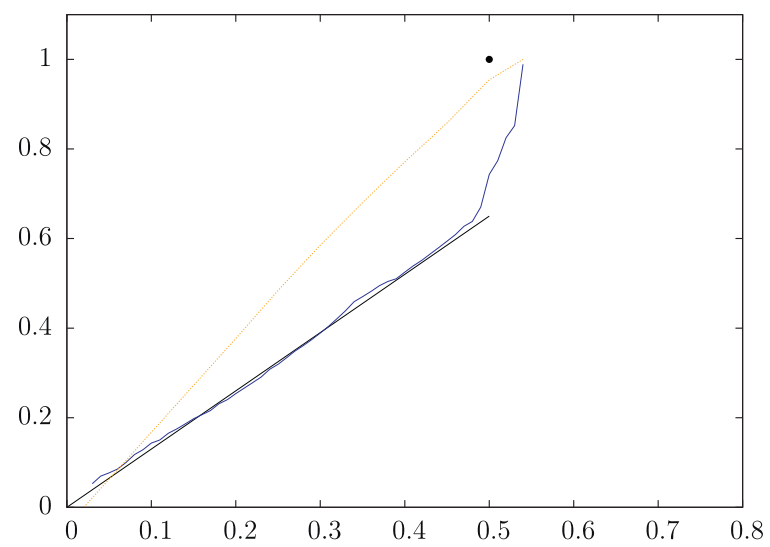

Fig. 9. Spectrum of a Lévy process which is the sum of a pure jump process $(\beta=1.3)$ and a Brownian motion $(H=0.5)$. Theoretical spectrum $(\cdots)$, WPM (_ ) and WLM spectrum (_-_). The results are the averages over one hundred realizations of length $2^{20}$.

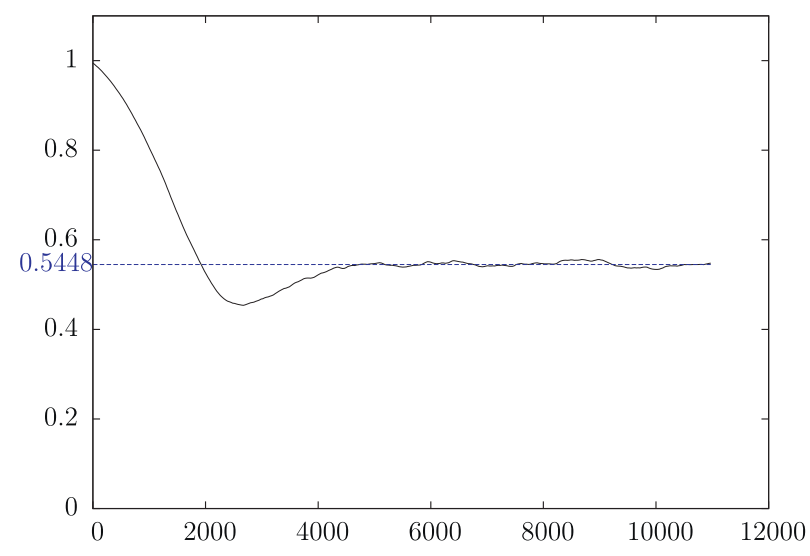

(a) $\alpha=0.4$ and $l=628.746$

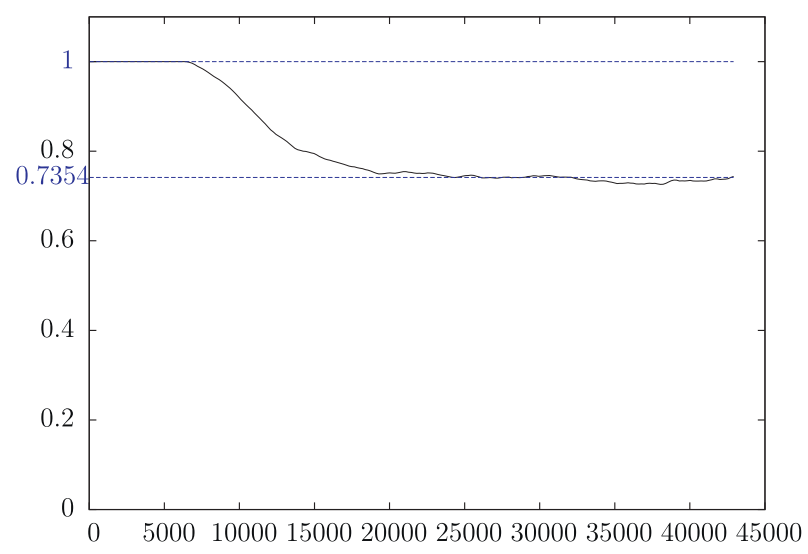

(b) $\alpha=0.52$ and $l=3605.66$

Fig. 10. Function $C_{\mapsto} \nu^{C}(\alpha)$ for a simulation of a Lévy process $(\beta=1.3)$ with a Brownian motion. We search a stabilization on an interval [ $C_{1}$, $\left.C_{2}\right]$ whose length $l$ depends on $\alpha$ (see Section 4.3).

For a fixed size $2^{j}(11 \leq j \leq 20)$, we have simulated one hundred Lévy processes with a Brownian part $(\beta=1.3)$. For each fixed $j$, we have computed the RMSE of each simulation. The boxplot and the mean of these RMSE are represented in Fig. 11. The WPM is obviously better than the WLM. However let us remark that for the signals of size $2^{11}$, it is very difficult to have an acceptable stabilization for the function $C \mapsto v_{f}^{C}(\alpha)$ for almost every $\alpha$. We have less than $15 \%$ of the signals with an acceptable stabilization; it is thus impossible to represent the boxplot of these signals for the WPM.

\subsection{Multifractal examples with non-concave spectra: superposition of two log-normal cascades}

Our last example is obtained by superposing two log-normal cascades. More precisely, if $m_{1}$ and $m_{2}$ are two measures associated with a log-normal cascade, we define the measure $m$ by

$$
m=\tilde{m}_{1}+\tilde{m}_{2}
$$

where $\tilde{m}_{1}$ (resp. $\left.\tilde{m}_{2}\right)$ is a measure defined on $[0,1 / 2](\operatorname{resp} .[1 / 2,1])$ such that $\tilde{m}_{1}(I)=m_{1}(2 I)\left(\operatorname{resp} . \tilde{m}_{2}(I)=m_{2}(2(I-1 / 2))\right.$. Clearly, the spectrum is given by

$$
d_{m}(h)=\sup \left\{d_{m_{1}}(h), d_{m_{2}}(h)\right\} .
$$

In Fig. 12, we compare the spectra obtained via the WPM and the WLM. The second method can only compute the concave hull of the theoretical spectrum; it is thus impossible to detect the presence of two log-normal cascades. With this method, one could conclude that there is only one cascade in the signal. On the other hand, the WPM shows the existence of a second phenomenon in the signal.

For a fixed size $2^{j}(11 \leq j \leq 20)$, we have simulated one hundred superpositions of two log-normal cascades and have computed the RMSE of each simulation of size $2^{j}$. The boxplot and the mean of these RMSE are represented in Fig. 13. We 


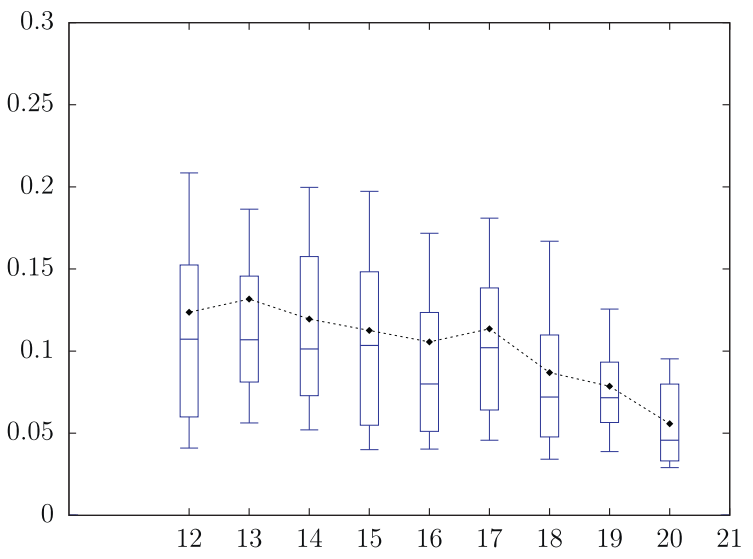

(a) WPM

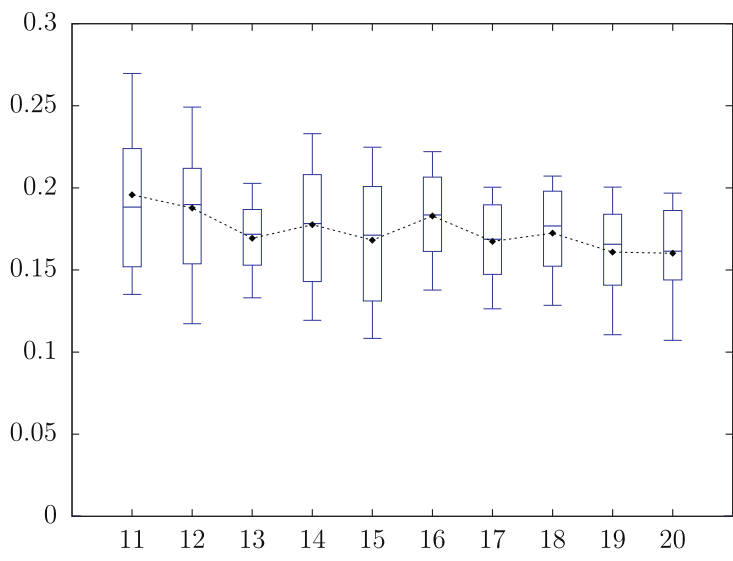

(b) WLM

Fig. 11. Functions that represent for every $j$ the boxplot of the RMSE of one hundred Lévy processes which are the sum of a pure jump process ( $\beta=1.3$ ) and a Brownian motion $(H=0.5)$ of size $2^{j}$. The mean of these RMSE is also represented (in black).

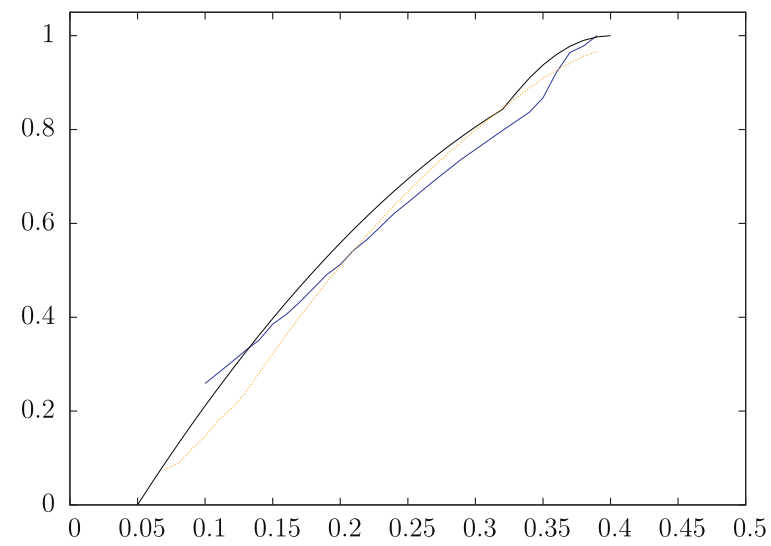

Fig. 12. The increasing part of the spectrum of the superposition of two log-normal cascades with parameters $\mu_{1}=-0.45 \log 2, \sigma_{1}^{2}=\sqrt{0.1 \log 2}, \mu_{2}=$ $-0.33 \log 2$ and $\sigma_{2}^{2}=0.02 \log 2$ where the first (resp. second) cascade is shifted to the right by 0.1 (resp. by 0.05 ). Theoretical spectrum ( $\cdots$ ), $S^{\nu}$ spectrum (_ ) and WLM spectrum (__ ). The results are obtained with a realization of length $2^{20}$.

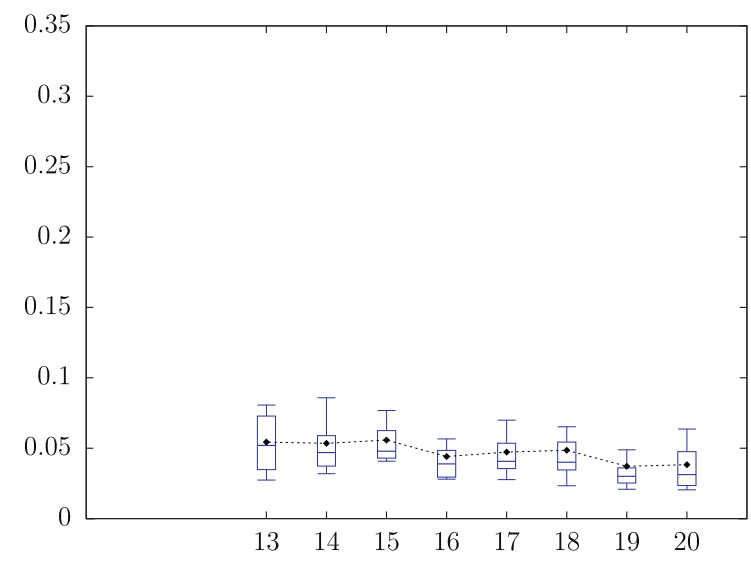

(a) WPM

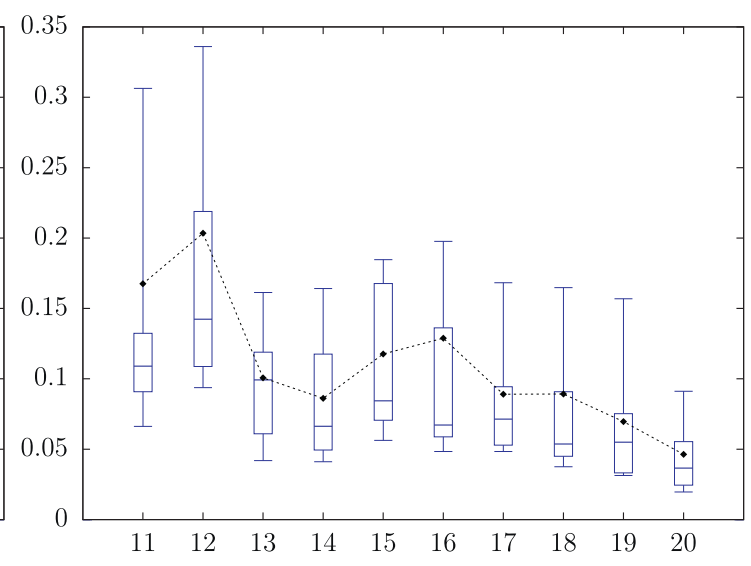

(b) WLM

Fig. 13. Functions that represent for every $j$, the boxplot of the RMSE of one hundred log-normal cascades with parameters $\mu_{1}=-0.45 \log 2, \sigma_{1}^{2}=$ $\sqrt{0.1 \log 2}, \mu_{2}=-0.33 \log 2$ and $\sigma_{2}^{2}=0.02 \log 2$ where the first (resp. second) cascade is shifted to the right by 0.1 (resp. by 0.05 ) of size $2^{j}$. The mean of these RMSE is also represented (in black). 


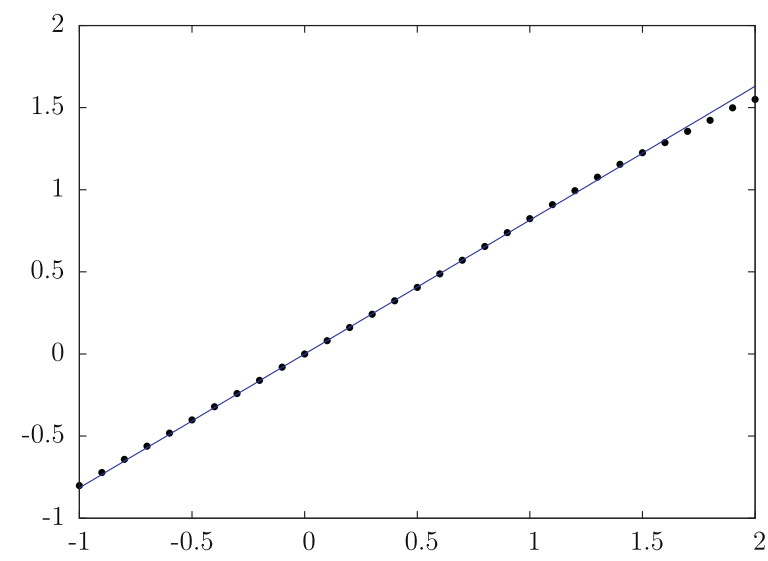

(a) the slope equals 0.815168

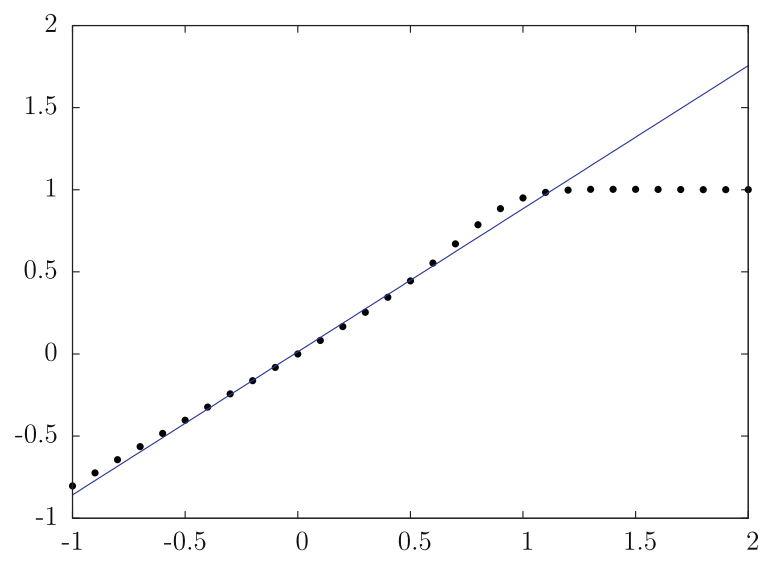

(b) the slope equals 0.871209

Fig. 14. Function $q_{\mapsto} \eta_{f}(q)$. Right (resp. left) for the fBm (resp. abfBm) of size $2^{15}$ with $H=0.8$.

clearly see that the superiority of the WPM over the WLM. Let us remark that, as for the Lévy process, for the size $2^{11}$ and $2^{12}$, less than $10 \%$ of the signals give an acceptable stabilization for the function $C \mapsto v_{f}^{C}(\alpha)$ for almost any $\alpha<0.34$. In these cases, the WLM leads to a spectrum, but it is not a good approximation of the theoretical spectrum. The boxplot associated to these signals for the WLM are also clearly worse than the others.

\subsection{Robustness of the method: aberrant values in fractional Brownian motions}

In practice, aberrant values can appear in a signal (for example, see [4]). These values can influence the analysis of the signal. This is illustrated in this section with the fractional Brownian motion (denoted by $\mathrm{fBm}$ ) where one value of the signal is modified. More precisely, one point of the signal is replaced by four times the largest value of the signal (such a modification is inspired by real-life signals [4]). This signal will be denoted by afBm.

Let us recall that the WLM approximates the Hölder exponent of a monofractal signal by the slope of the function $q \mapsto \eta_{f}(q)$ where

$$
\eta_{f}(q)=\liminf _{j \rightarrow+\infty} \frac{2^{-j} \sum_{k}\left|d_{j, k}\right|^{q}}{\log 2^{-j}}
$$

where $\left(d_{j, k}\right)_{j, k}$ denote the wavelet leaders of the signal ; they are defined as local suprema of wavelet coefficients, see [57] for more details.

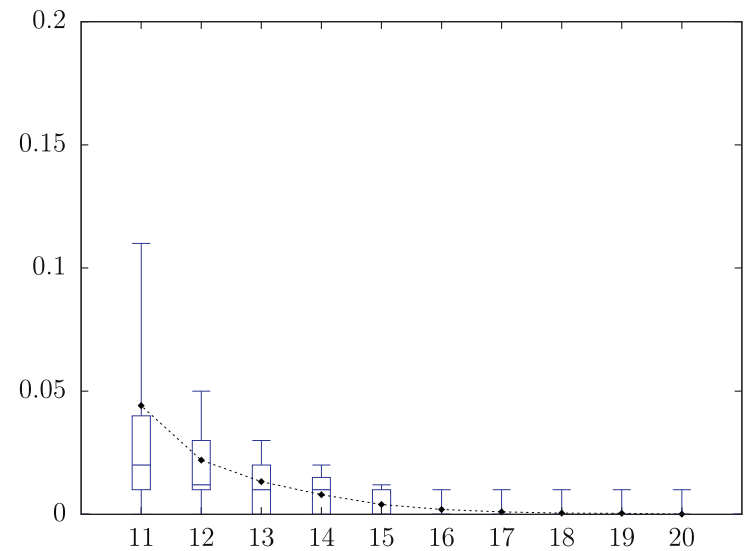

(a) WPM

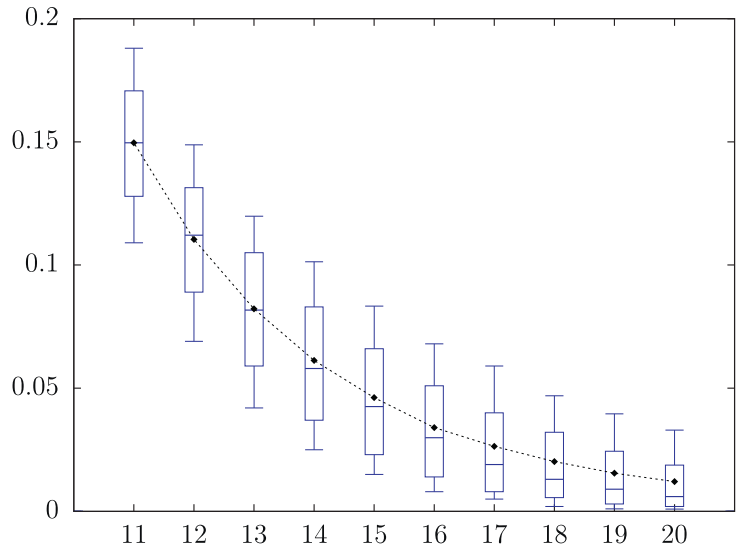

(b) WLM

Fig. 15. Functions that represent for every $j$, the boxplot of the distances between the Hölder exponent detected for the fBm and the exponent detected for the corresponding afBm of size $2^{j}$. The mean of these distances is also represented (in black). 
For the fBm, this method gives good results (see Section 5.1) but the function $\eta_{f}$ is strongly disturbed with the afBm, as illustrated in Fig. 14. Indeed, such a modification of the $\mathrm{fBm}$ implies that, at each scale $j$, at least one wavelet coefficient is strongly modified. As a result the function $\eta_{f}$ has a flat part for the largest values of $q$ and the slope of the increasing part does not give a correct approximation of the Hölder exponent. The WPM (resp. WLM) approximates the Hölder exponent by 0.81 and 0.82 (resp. 0.81 and 0.87 ) for the $\mathrm{fBm}$ and the afBm respectively.

Fig. 15 shows for every $j$, the mean and the boxplot of the distance between the Hölder exponent detected for the fBm and the exponent detected for the corresponding afBm using the WPM and the WLM. The robustness of the WPM is clear. For the sizes $2^{j}$ with $15 \leq j \leq 20$, this distance is smaller than 0.01 . For smaller sizes, this distance is also very small, excepted for a few signals of size $2^{11}$. On the other hand, the WLM is very disturbed. For the sizes $2^{j}$ with $16 \leq j \leq 20$, the distance has the same order of magnitude as the approximation of the exponent of the original signal (see Fig. 4). For the smaller sizes, the exponent detected for the afBm is significantly different from the true exponent.

\section{Conclusion}

We have introduced an algorithm that allows to apply the $S^{\nu}$-based multifractal formalism to numerical data. Similar methods have been proposed [27], but the implementation described in Section 4 is the only one which is stand-alone.

For monofractal and concave spectra, our method gives similar results to the ones obtained using the wavelet leaders method for the increasing part of the spectrum. Moreover, the WPM leads to the detection of non-concave spectra, where, as already known, the wavelet leaders method fails and only leads to the concave hull of such spectra.

However, the WPM may be unsuccessful in detecting the spectrum for the smallest values of $h$. This is due to the fact that more coefficients are needed to properly compute the $S^{v}$ spectrum.

Moreover, the WPM is more robust to aberrant values, as shown in Section 6.3.

\section{Acknowledgments}

The second author is supported by the Labex CEMPI (ANR-11-LABX-0007-01).

\section{Appendix A}

In practice, the choice of the constant $C$ used to compute an approximation of the value $v(\alpha)$ is very important; the computed value $v^{C}(\alpha)$ is dependent of $C$ and can be very different from the theoretical value $v(\alpha)$. Our approach is not to find a constant $C$ but rather an interval where the value $v^{C}(\alpha)$ is (nearly) constant. In a previous article [44], the length of this interval was taken to be equal to the greatest value among the moduli of the wavelet coefficients. In what follows, this approach is called method 1 and the approach of this article is called method 2. This appendix illustrates a few problems of method 1 and shows that method 2 solves them.

First, as illustrated in Fig. 16, method 1 is less efficient for the smallest sizes of the signal than method 2.

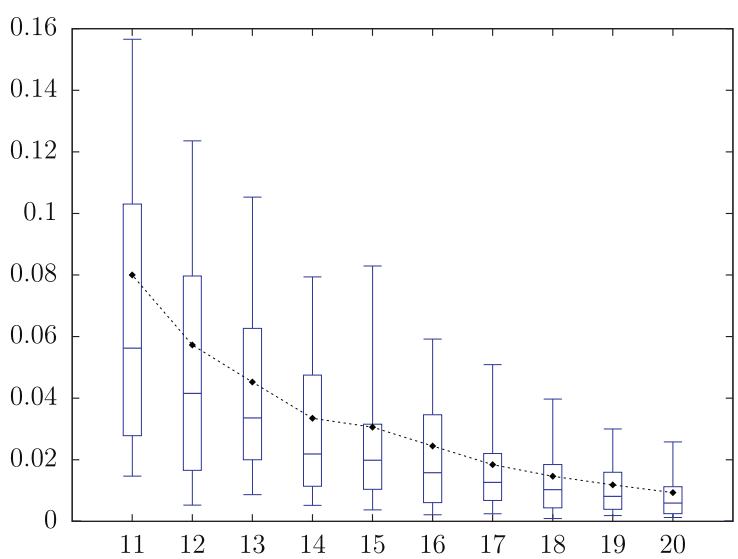

(a) method 1

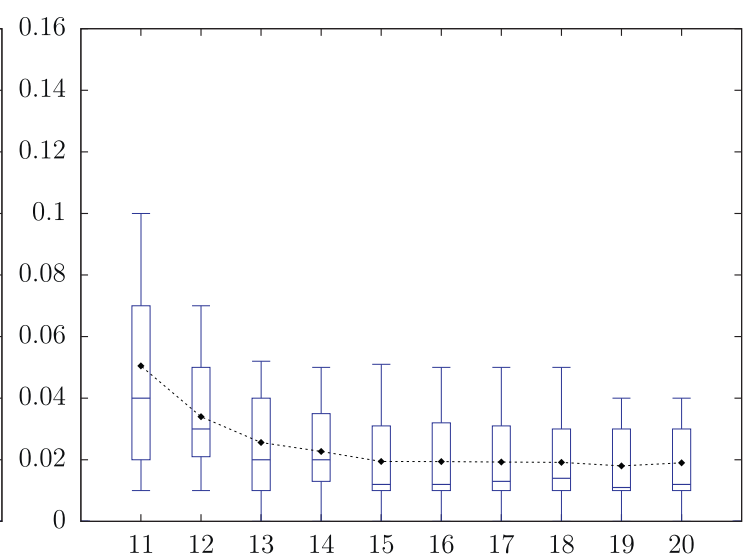

(b) method 2

Fig. 16. Functions that represent for every $j$, the boxplot of the distances between the Hölder exponent detected and $H$ (for $H$ which varies between 0.2 and 0.8 by steps of 0.05 ) for walks of fractional Brownian motion of size $2^{j}$ with parameter $H$. The mean of these distances is also represented (in black). 


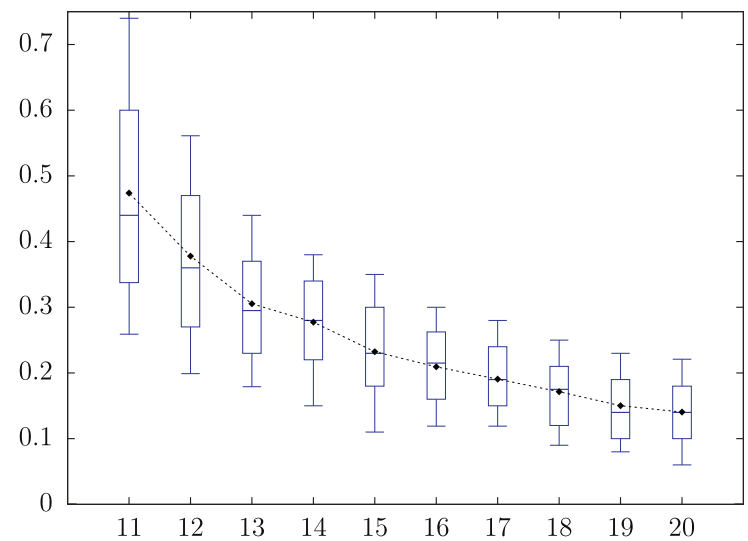

(a) method 1

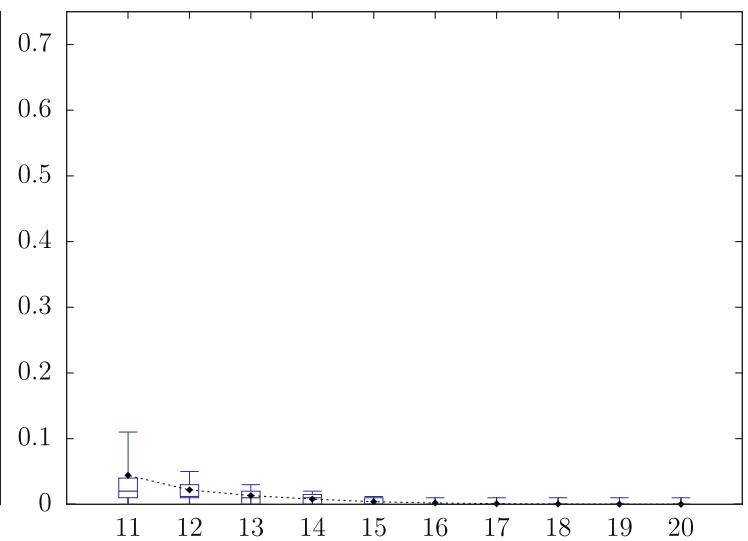

(b) method 2

Fig. 17. Functions that represent for every $j$, the boxplot of the distances between the Hölder exponent detected for the fBm and the exponent detected for the corresponding afBm of size $2^{j}$. The mean of these distances is also represented (in black).

Table 1

The median value for the smallest $h$ detected for a Lévy process of size $2^{j}$ ( $\beta=$ $1.3)$.

\begin{tabular}{llll}
\hline & Method 1 & Method 2 & Difference between the two methods \\
\hline$j=20$ & 0.09 & 0.26 & 0.17 \\
$j=19$ & 0.13 & 0.27 & 0.14 \\
$j=18$ & 0.19 & 0.27 & 0.08 \\
$j=17$ & 0.18 & 0.28 & 0.1 \\
$j=16$ & 0.19 & 0.3 & 0.11 \\
$j=15$ & 0.2 & 0.33 & 0.13 \\
$j=14$ & 0.22 & 0.34 & 0.12 \\
$j=13$ & 0.3 & 0.37 & 0.07 \\
$j=12$ & 0.36 & 0.43 & 0.07 \\
$j=11$ & 0.45 & 0.56 & 0.11 \\
\hline
\end{tabular}

Moreover, it is easy to convince oneself that method 1 is strongly influenced by aberrant values in the signal, as for the WLM, since the size of the interval in this case is given by the largest wavelet coefficient. In Fig. 17, we compare the two methods for the afBm (as in Section 6.3): we clearly see the influence of an aberrant value in the signal in method 1.

Finally, method 2 allows to approximate the spectrum for smaller values of $h$ compared to method 1 . Table 1 gives the median value for the smallest detected $h$ for a Lévy process of size $2^{j}$. We clearly see the advantages of the method proposed in this article.

\section{References}

[1] Muzy JF, Bacry E, Arneodo A. Wavelets and multifractal formalism for singular signals: application to turbulence data. Phys Rev Lett 1991;67:3515-18. doi:10.1103/PhysRevLett.67.3515.

[2] Pavlov A. N., Pavlova O. N., Abdurashitov A. S., Arinushkin P. A., Runnova A. E., Semyachkina-Glushkovskaya O. V.. Multifractal spectrum of physiological signals: a mechanism-related approach. 2017. doi: 10.1117/12.2267692.

[3] Nicolay S, Brodie of Brodie EB, Touchon M, Audit B, d'Aubenton Carafa Y, Thermes C, et al. Bifractality of human DNA strand-asymmetry profiles results from transcription. Phys Rev E 2007;75:032902. doi:10.1103/PhysRevE.75.032902.

[4] Deliège A, Kleyntssens T, Nicolay S. Mars topography investigated through the wavelet leaders method: a multidimensional study of its fractal structure. Planet Space Sci 2017;136:46-58. doi:10.1016/j.pss.2016.12.008.

[5] Mandelbrot B. Fractals: form, chance, and dimension. W.H. Freeman; 1977.

[6] Mandelbrot B. The fractal geometry of nature. Freeman and Co.; 1982.

[7] Jaffard S. Old friends revisited: the multifractal nature of some classical functions. J Fourier Anal Appl 1996;3:1-22.

[8] Jaffard S. Multifractal formalism for functions part I: results valid for all functions. SIAM J Math Anal 1997;28:944-70

[9] Arneodo A, Audit B, Decoster N, Muzy J-F, Vaillant C. Climate disruptions, market crashes, and heart attacks. In: Bunder A, Schellnhuber H, editors. The science of disaster. Springer; 2002. p. 27-102.

[10] Jaffard S, Nicolay S. Pointwise smoothness of space-filling functions. Appl Comput Harmon Anal 2009;26:181-99.

[11] Jaffard S, Nicolay S. Space-filling functions and Davenport series. In: Recent developments in fractals and related fields. Springer; 2010. p. 19-34.

[12] Clausel M, Nicolay S. Wavelets techniques for pointwise anti-Hölderian irregularity. Constr Approx 2011;33:41-75.

[13] Falconer K. The geometry of fractal sets. Cambridge University Press; 1986. ISBN 9780521337052.

[14] Hardy G. Weierstrass's non-differentiable function. Trans Am Math Soc 1916;17:301-25.

[15] Testud B. Étude d'une classe de mesures autosimilaires: calculs de dimensions et analyse multifractale, Ph.D. thesis; 2004

[16] Parisi G, Frisch U. On the singularity structure of fully developed turbulence. Turbul Predict Geophys Fluid Dyn 1985:84-7.

[17] Arneodo A, Grasseau G, Holschneider M. Wavelet transform of multifractals. Phys Rev Lett 1988;61:2281-4. 
[18] Jaffard S. On the Frisch-Parisi conjecture. J Math Pures Appl 2000;79:525-52.

[19] Arneodo A, Bacry E, Muzy J-F. The thermodynamics of fractals revisited with wavelets. Physica A 1995;213:232-75.

[20] Jaffard S. Multifractal formalism for functions part II: self-similar functions. SIAM J Math Anal 1997;28:971-98.

[21] Slimane MB. Multifractal formalism and anisotropic selfsimilar functions. Math Proc Camb Philos Soc 1998;124:329-63.

[22] Jaffard S. Beyond Besov spaces part 2: oscillation spaces. Constr Approx 2005;21:29-61.

[23] Jaffard S. Wavelet techniques in multifractal analysis, fractal geometry and applications: a jubilee of Benoit Mandelbrot. Proc Symp Pure Math 2004; 72:91-151.

[24] Muzy J-F, Bacry E, Arneodo A. Multifractal formalism for fractal signals: the structure function approach versus the wavelet-transform mudulus-maxima method. Phys Rev E 1993;47:875-84.

[25] Jaffard S. Beyond Besov spaces part 1: distributions of wavelet coefficients. J Fourier Anal Appl 2004;10:221-46.

[26] Aubry J-M, Bastin F, Dispa S, Jaffard S. Topological properties of the sequence spaces $S^{\nu}$. J Math Anal Appl 2006;1:364-87.

[27] Abry P, Jaffard S, Wendt H. A bridge between geometric measure theory and signal processing: multifractal analysis. Cham: Springer International Publishing; 2015. p. 1-56. ISBN 978-3-319-08557-9.

[28] Aubry J-M, Bastin F, Dispa S, Jaffard S. Prevalence of multifractal functions in $S^{v}$ spaces. J Fourier Anal Appl 2007;13:175-85.

[29] Christensen J. On sets of haar measure zero in abelian polish groups. Israel J Math 1972;13:255-60.

[30] Hunt BR, Sauer T, Yorke JA. Prevalence: a translation-invariant “almost every" on infinite-dimensional spaces. Bull Am Math Soc 1992;27(2):217-38. doi:10.1090/S0273-0979-1992-00328-2.

[31] Daubechies I. Ten lectures on wavelets. CBMS-NSF Regional Conference Series in Applied Mathematics. SIAM: Society for Industrial and Applied Mathematics; 1992.

[32] Meyer Y, Salinger D. Wavelets and operators, 1. Cambridge University Press; 1995.

[33] Mallat S. A wavelet tour of signal processing. Academic Press; 1999. ISBN 0-12-466606-X.

[34] Jaffard S. Pointwise smoothness, two-microlocalization and wavelet coefficients. Publicacions Matemtiques 1991;35(1):155-68.

[35] Aubry J-M, Jaffard S. Random wavelet series. Commun Math Phys 2002;227:483-514.

[36] Aubry J-M, Bastin F. A walk from multifractal analysis to functional analysis with $S^{v}$ spaces, and back. In: Recent developments in fractals and related fields. Springer; 2010. p. 93-106.

[37] Meneveau C, Sreenivasan KR. Simple multifractal cascade model for fully developed turbulence. Phys Rev Lett 1987;59:1424-7. doi:10.1103/ PhysRevLett.59.1424.

[38] Barral J, Seuret S. From multifractal measures to multifractal wavelet series. J Fourier Anal Appl 2005;11(5):589-614.

[39] Seuret S. Multifractal analysis and wavelets. In: New trends in harmonic analysis. Birkhäuser; 2013. Lecture notes from the CIMPA school

[40] Lévy Véhel J, Vojak R. Multifractal analysis of choquet capacities. Adv Appl Math 1998;20(1):1-43.

[41] Mallat S. Multiresolution approximations and wavelets. Trans Am Math Soc 1989;315:69-87.

[42] Daubechies I. Orthonormal bases of compactly supported wavelets. Commun Pure App Math 1988;41:909-96.

[43] Snyman J. Practical mathematical optimization. Applied optimization, 97. Springer; 2005.

[44] Esser C, Kleyntssens T, Nicolay S. A multifractal formalism for non-concave and non-increasing spectra: the leaders profile method. Appl Comput Harmon Anal 2016;43(2):269-91.

[45] Maman D. Généricité et prévalence des propriétés multifractales de traces de fonctions; 2013. Ph.D. thesis.

[46] Mandelbrot B, van Ness J. Fractional Brownian motions, fractional noises and applications. SIAM Rev 1968;10:422-37.

[47] Comte F, Renault E. Long memory in continuous-time stochastic volatility models. Math Financ 1998;8:291-323.

[48] Audit B, Thermes C, Vaillant C, d'Aubenton Carafa Y, Muzy J-F, Arneodo A. Long-range correlations in genomic DNA: a signature of the nucleosomal structure. Phys Rev Lett 2001;86:2471.

[49] Nicolay S, Touchon M, Audit B, d'Aubenton Carafa Y, Thermes C, Arneodo A, et al. Bifractality of human DNA strand-asymmetry profiles results from transcription. Phys Rev E 2007;75:032902.

[50] Kahane J.-P.. Some random series of functions. 1995.

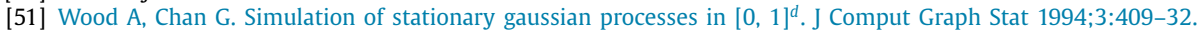

[52] Mandelbrot B. Intermittent turbulence in self-similar cascades: divergence of high moments and dimension of the carrier. J Fluid Mech 1974;62:351-8.

[53] Arneodo A, Bacry E, Muzy JF. Random cascades on wavelet dyadic trees. J Math Phys 1998;39:4142-64.

[54] Raible S. Lévy processes in finance: theory, numerics, and empirical facts, Ph.D. thesis; 2000.

[55] Jaffard S. The multifractal nature of Lévy processes. Probab Theory Relat Fields 1999;114:207-27.

[56] Mantegna RN. Fast, accurate algorithm for numerical simulation of Lévy stable stochastic processes. Phys Rev E 1994;49:4677-83

[57] Jaffard S, Lashermes B, Abry P. Wavelet leaders in multifractal analysis. Basel: Birkhäuser; 2007. p. 201-46. ISBN 978-3-7643-7778-6. 